90

The Open Biomedical Engineering
Content list available at: https://openbiomedicalengineeringjournal.com
Biomedical
lournal

REVIEW ARTICLE

\title{
Essentials of Predicting Epileptic Seizures Based on EEG Using Machine Learning: A Review
}

\author{
Vibha Patel $^{1, *}$, Jaishree Tailor ${ }^{2}$ and Amit Ganatra ${ }^{3}$ \\ 'Department of Computer Engineering, Chhotubhai Gopalbhai Patel Institute of Technology (CGPIT), Uka Tarsadia University, Gujarat, India \\ ${ }^{2}$ Shrimad Rajchandra Institute of Management and Computer Application (SRIMCA), Uka Tarsadia University, Gujarat, India \\ ${ }^{3}$ Department of Computer Engineering, DEPSTAR, Charotar University of Science and Technology (CHARUSAT), Gujarat, India
}

\begin{abstract}
:
Objective:

Epilepsy is one of the chronic diseases, which requires exceptional attention. The unpredictability of the seizures makes it worse for a person suffering from epilepsy.

Methods:

The challenge to predict seizures using modern machine learning algorithms and computing resources would be a boon to a person with epilepsy and its caregivers. Researchers have shown great interest in the task of epileptic seizure prediction for a few decades. However, the results obtained have not clinical applicability because of the high false-positive ratio. The lack of standard practices in the field of epileptic seizure prediction makes it challenging for novice ones to follow the research. The chances of reproducibility of the result are negligible due to the unavailability of implementation environment-related details, use of standard datasets, and evaluation parameters.

Results:

Work here presents the essential components required for the prediction of epileptic seizures, which includes the basics of epilepsy, its treatment, and the need for seizure prediction algorithms. It also gives a detailed comparative analysis of datasets used by different researchers, tools and technologies used, different machine learning algorithm considerations, and evaluation parameters.

Conclusion:

The main goal of this paper is to synthesize different methodologies for creating a broad view of the state-of-the-art in the field of seizure prediction.
\end{abstract}

Keywords: Epilepsy, EEG, Machine learning, Seizure prediction, India, Behavioral, Health.

\begin{tabular}{|l|l|l|l|}
\hline Article History & Received: October 26, 2020 & Revised: April 20,2021 & Accepted: May 6, 2021 \\
\hline
\end{tabular}

\section{INTRODUCTION}

Epilepsy is a chronic non-communicable disease of the brain that affects people of all ages. According to the World Health Organization (WHO), around 50 million people suffer from epilepsy worldwide. It is estimated that there are more than 10 million people with epilepsy in India. Globally, an estimated five million people are diagnosed with epilepsy each year. Epilepsy leads to behavioral, health, and economic consequences. Efficient diagnosis and treatment are very much

\footnotetext{
* Address correspondence to this author at Department of Computer Engineering, Chhotubhai Gopalbhai Patel Institute of Technology (CGPIT), Uka Tarsadia University, Gujarat, India; E-mail: vibha.patel@utu.ac.in
}

essential for the quality of life of the person with epilepsy and its caregivers.

With the increasing computing availability and storage, machine learning algorithms have been widely explored in the area of epileptic seizure prediction. However, because of the diversified research approaches, dataset considerations, evaluation parameters, and implementation approaches, the reproducibility of the results is limited. The work presented here focuses on the essential aspects of epileptic seizure prediction. The future research approaches may consider the presented comparative analysis of datasets, implementation tools, and methodologies for evolved epileptic seizure prediction methodology. 
The organization of this paper is as follows: Section-2 describes the fundamentals of epilepsy and treatment. Section-3 and 4 describe the use of electroencephalogra-phy (EEG) for epileptic seizure prediction and the need for algorithms. Section- 5 gives the comparative analysis of various approaches for epileptic seizure detection and prediction, followed by Section-6 with the research gaps. Section-7 gives a detailed understanding of datasets used in prior research of epileptic seizure prediction. Section- 8 gives a comparative analysis of tools and libraries used for the implementation of epileptic seizure prediction systems, which is followed by the conclusion.

\section{EPILEPSY AND TREATMENT}

Seizures are due to excessively synchronous and/or excessively intense activity of neuronal circuits in the brain, particularly in the cerebral cortex. In epilepsy, seizures occur spontaneously, repeatedly, and usually suddenly. The unpredictability of seizures represents one of the main disabling features of epilepsy [1]. To date, the causes of epilepsy have not been identified. However, conditions like severe head injury, stroke and blood vessel diseases, tumors, changes in brain structure, and brain infections could provoke seizures [2]. Epileptic seizures can be classified into three groups [3]. Generalized Onset Seizures: These seizures affect both sides of the brain or groups of cells on both sides of the brain at the same time. This term still includes seizure types like tonic-clonic, absence, or atonic. Focal Onset Seizures: It can start in one area or group of cells on one side of the brain. Unknown Onset Seizures: When the beginning of a seizure is not known, it is called an unknown onset seizure.

Epileptic seizures have four different states: the preictal state, which is a state that appears before the seizure begins, the ictal state that begins with the onset of the seizure and ends with an attack; the postictal state that starts after ictal state, and interictal state that starts after the postictal state of first seizure and ends before the start of the preictal state of consecutive seizure [4]. The effect of epilepsy is different in each individual. So, recognizing and diagnosing the type of seizure or epilepsy affecting a person can sometimes be challenging. However, there are a few common ways of testing and determining an epilepsy diagnosis [3]. Various methods, like electroencephalography (EEG), Computerized Tomography (CT) scans, Magnetic Resonance Imaging (MRI), and functional imaging studies, are used to evaluate a person with epilepsy $[5,6]$. EEG is the most common method amongst all for the diagnosis and treatment of epilepsy. Persons with epilepsy are generally treated with Anti-Epileptic Drugs (AEDs). A high dosage of AEDs optimally controls epileptic seizures. However, regular consumption of AEDs generally shows side effects like tiredness, headache, dizziness, or blurred vision. It may also lead to behavioral changes in the person with epilepsy [7]. About $20-40 \%$ of persons with epilepsy are drug-resistant, i.e., they do not respond to AEDs even though a variety of drugs are available for decades $[8,9]$, of whom only a small minority can be helped by epilepsy surgery [10].

\section{ELECTROENCEPHALOGRAPHY(EEG)}

An electroencephalogram (EEG) is the flow of neuronal ionic currents recorded using a pair of electrodes either inside or outside the scalp [11]. The applications of EEG signal processing are brain-computer interface, seizure detection, seizure prediction, schizophrenia detection and classification, diagnosis of Parkinson's disease, etc. EEG is significantly used in the diagnosis, classification, and treatment of epileptic seizures [8]. If an invasive technique is used to record the EEG signal from inside the skull, it is called intracranial EEG (iEEG). In a non-invasive technique, EEG signals are recorded from the scalp, called scalp EEG (sEEG). EEG waveforms are generally classified into normal and abnormal signals using frequency parameters [12]. EEG signals can be categorised into the following based on frequency: Delta $(0.1-4 \mathrm{~Hz})$, Theta (4 $8 \mathrm{~Hz})$, Alpha (8- $13 \mathrm{~Hz})$, Beta (13 - $30 \mathrm{~Hz})$, and Gamma (30 $100 \mathrm{~Hz}$ ). Different EEG frequency corresponds to different behavior and mental state of the brain [12]. Due to the high complexity of EEG signals, a single prediction feature can only quantify some of its properties [7].

The popularity of EEG is because of the following advantages: scalp EEG (sEEG) is a non-invasive technique which records the waveforms without much effort or active response by the subject/patient, EEG recording kit is portable and financially affordable, EEG recording devices do not make any noise and no special environment is needed to set it up [11]. Though EEG techniques are widely evolved, there are still a few limitations that need to be addressed. Such as, EEG is prone to low spatial resolution and low signal-to-noise ratio (SNR) [11]. Preprocessing of EEG is also very challenging. To have an artefact-free EEG to extract the control signals, the EEGs have to be restored from the artefacts, such as eyeblinking, electrocardiograms (ECGs), and any other internal or external disturbing effects [13].

\section{THE NEED FOR EPILEPTIC SEIZURE PREDICTION ALGORITHMS}

With the evolving EEG technology and resource advances, there has been huge interest in the EEG waveform-based research for brain-computer interface (BCI), disease detection, and treatment. Characterization of EEG waveforms plays a vital role in the field of epileptic seizure detection and classification. With the use of sEEG or iEEG signals, certain patterns can be found to detect the preictal state of seizure. The detection of a preictal state would trigger an alarm for the patient or patient's caregivers to take precautions or medicine beforehand to avoid the ill effect of seizure [7]. Seizure detection methods can be used for the offline analysis of EEG waveforms or seizure-abortion devices. Whereas the seizure prediction system identifies the occurrence of seizure before a certain period called the occurrence period [7].

With rapidly increasing computing power and storage, the availability of EEG data has become easier. Researchers have started using the power of modern machine learning algorithms to improve the results of seizure prediction algorithms [14 27]. Though ample research has already been done, there is no clinical applicability yet $[28,29]$. This is because of the sensitivity of the seizure prediction algorithms. One of the 
challenges in the study of epileptic seizure detection and prediction is to achieve the results to apply the same for clinical applicability [7]. The statistical justification is also desirable for a complete understanding of existing approaches that achieve significant results [7]. For clinical applicability of seizure prediction approaches, the alarm shall trigger prior to a considerable time period. So, the seizure prediction horizon needs to be one of the important evaluation parameters of such systems [7]. Researchers have incorporated various approaches for epileptic seizure prediction. The following sections review the datasets incorporated, tools and libraries used and different algorithms considered for the same. This would help novice ones to explore and improve machine learning-based epileptic seizure prediction algorithms.

\section{MACHINE LEARNING ALGORITHMS AND EVALUATION PARAMETERS}

The work for epilepsy seizure prediction has evolved drastically since its inception. Based on various methodologies followed and algorithms used, epilepsy seizure prediction can be categorized into four approaches as follows. Also, Fig. (1) shows all four approaches:

[1] The traditional machine learning approach

[2] Deep learning approach

[3] Signal processing approach

[4] Hybrid approach
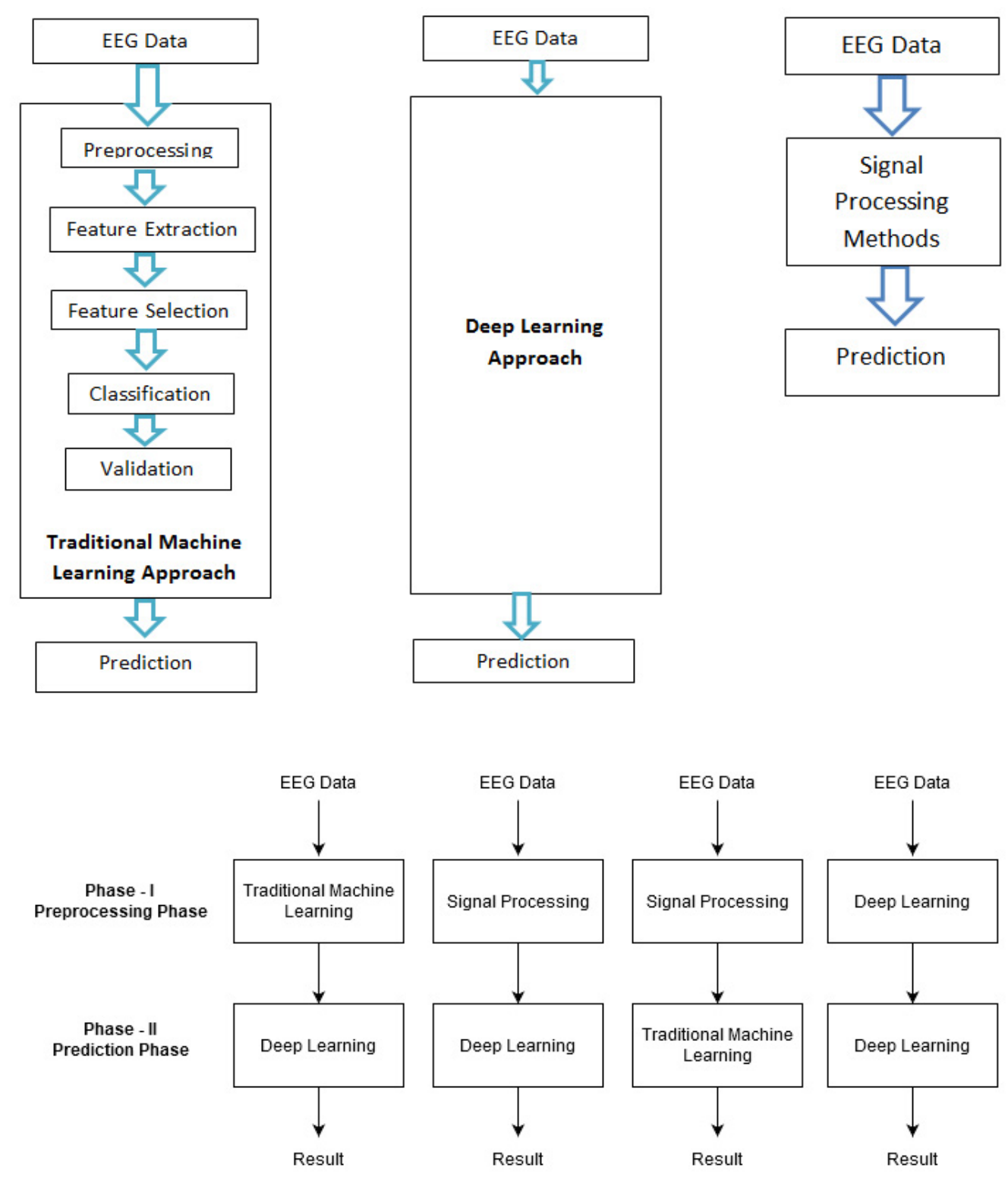

Fig. (1). Diagrammatic representation of commonly used approaches for epileptic seizure prediction. 


\subsection{The Traditional Machine Learning Approach}

The traditional machine learning approach includes various stages like preprocessing, feature extraction, feature selection, classification, and validation. Handcrafted features are being used for the detection of the preictal state. A brief description of all the stages is as follows: Data Cleaning, EEG data is nonstationary and prone to artefacts, it requires high preprocessing. This is the phase that takes maximum effort to read, interpret, and clean the noise. Feature Extraction, using different signal processing techniques, features are extracted in time domain, frequency domain, or time and frequency domain. Feature Selection, a huge amount of features is being generated from the feature extraction phase. It is important to find the feature of importance for a specific task. Some of the features considered by researchers are entropy, approximate entropy, Hjorth parameters, spectral moments, mobility, energy, entropy, correlation coefficients, Fast Fourier Transform (FFT), variance, skewness, kurtosis, mean, fractal dimension, frequency band power, peak amplitude, zero crossing, average spectral power, line length, and maximal and minimal values. Classification, the problem of epileptic seizure prediction and detection is actually to differentiate the pre-ictal state of EEG signal from the interictal and ictal state. Different classification algorithms like Support Vector Machine, knearest neighbor, Gaussian Naive Bayes, random forest, multilayer perceptron are used for this purpose, Validation, the hyperparameters are not trained by classification models, it would be predefined. Model performance depends highly on the selection of hyperparameters. The best model amongst various values of hyperparameters is chosen in the validation stage. Prediction is the last phase that performs the task of prediction based on the optimal performing model. Table 1 gives the detailed comparative analysis of various studies conducted using the traditional machine learning approach for epileptic seizure state detection and prediction.

\subsection{Deep Learning Approch}

Deep learning-based models are end-to-end models, i.e. once EEG signals are given as input, an automated approach for feature extraction and selection is done. Based on the learning and validation, the model would converge and give the prediction results. Another noticeable approach for epileptic seizure prediction is based on signal processing methods. The large amount of data recorded from even a single EEG electrode pair presents a difficult interpretation challenge. Convolutional Neural Network (CNN), Recurrent Neural Network (RNN), and variations of these algorithms have been used by researchers in the past for the task of seizure prediction. This approach is trending because of the availability of resources. Table 2 gives the detailed comparative analysis of various studies conducted using the deep learning approach for epileptic seizure state detection and prediction.

\subsection{Signal Processing Approach}

In this approach, traditional signal processing methods are used to highly process the data. Signal processing methods are needed to automate signal analysis and interpret the signal phenomena [30]. The prediction stage of this approach uses a basic classification algorithm for signal state classification. Table 3 gives the detailed comparative analysis of various studies conducted using the signal processing approach for epileptic seizure state detection and prediction.

\subsection{Hybrid Approach}

The combination of all three methods, i.e., traditional machine learning, deep learning, and signal processing leads to a hybrid approach, which takes best of all. Literature provides the data related to the combination of traditional machine learning and deep learning models, deep learning, and signal processing models, machine learning and signal processing methods deep learning methods. References for some hybrid approaches provides the detailed comparative analysis of various studies conducted using the hybrid approach to epileptic seizure state detection and prediction.

To evaluate the performance of various models, different evaluation parameters like sensitivity, specificity, false-positive rate, accuracy, prediction time, AUC, ROC, and F1 score have been considered in the past by other researchers. However, there is no clear consensus regarding which parameter signifies the performance of epileptic seizure detection or prediction algorithms best. Yannick Roy et al. [31] and Alexander Craik et al. [32] have presented a vast comparison of all work done in the field of epileptic seizure prediction. Along with the comparison, various aspects for future research are also mentioned.

\section{RESEARCH GAPS}

To increase the reliability of seizure prediction results and clinical applicability of the same, evolved models of EEG signal analysis are the need of the time [11]. This section describes some of the improvement areas which could aid in the performance of seizure detection and prediction models, which may lead to clinical applicability. No methods have exhibited both high sensitivity and zero false alarms per hour to achieve the reliability of results [28]. Also, existing machine learning algorithms unnecessarily reduce the number of parameters in feature selection for simplistic classification [28]. The advanced techniques can be used for preprocessing of EEG data to get increased sensitivity of the results [4]. Identification of proper matrices for the evaluation of seizure prediction model is also a big challenge for highly imbalanced data. Most of the work done in the field of epileptic seizure detection and prediction focuses on patient-specific approaches. The concept of domain adaptation and transfer learning can be used for cross-patient research, i.e. generalized models [28]. A better generalization performance between subjects will be necessary to truly make BCIs useful [31]. All prediction methods have been developed and tested on different EEG data pools, making it difficult to compare their performance [101, 119]. 
Table 1. Comparative analysis of the traditional machine learning approach.

\begin{tabular}{|c|c|c|c|c|c|c|c|}
\hline Year & $\begin{array}{l}\text { Research } \\
\text { Group }\end{array}$ & Dataset Used & Preprocessing & Feature Extraction & $\begin{array}{l}\text { Feature } \\
\text { Selection }\end{array}$ & Classifier Used & Performance \\
\hline 2009 & $\begin{array}{c}\text { Theoden Netoff } \\
\text { et al. }[33]\end{array}$ & $\begin{array}{l}\text { European } \\
\text { Epilepsy } \\
\text { Database }\end{array}$ & $\begin{array}{c}\text { Removing artefacts, } \\
\text { filtering }\end{array}$ & Power of spectral bands & $\mathrm{N} / \mathrm{M}$ & $\begin{array}{c}\text { Cost-effective } \\
\text { SVM }\end{array}$ & \begin{tabular}{|c|} 
Sensitivity $=77.8 \%$ \\
False positive rate \\
per hour $=0$
\end{tabular} \\
\hline 2013 & $\begin{array}{c}\text { Ning Wang et al. } \\
{[34]}\end{array}$ & $\begin{array}{l}\text { European } \\
\text { Epilepsy } \\
\text { Database }\end{array}$ & $\mathrm{N} / \mathrm{M}$ & $\begin{array}{c}\text { Averaged Instantaneous } \\
\text { Envelope (AIE), Averaged } \\
\text { Instantaneous Frequency } \\
\text { (AIF) }\end{array}$ & $\begin{array}{l}\text { RFE-SVM } \\
\text { (Recursive } \\
\text { feature } \\
\text { elimination - } \\
\text { SVM) }\end{array}$ & SVM & $\begin{array}{c}\text { Average Sensitivity } \\
=98.8 \% \\
\text { Average False } \\
\text { alarm per hour }= \\
0.054 \\
\text { AUC }=0.784\end{array}$ \\
\hline 2014 & $\begin{array}{c}\text { Peyvand } \\
\text { Ghaderyana et } \\
\text { al. }[35]\end{array}$ & $\begin{array}{l}\text { European } \\
\text { Epilepsy } \\
\text { Database }\end{array}$ & $\begin{array}{c}\text { Removing artefacts, } \\
\text { normalized power }\end{array}$ & $\begin{array}{l}\text { Spectral bands, statistical } \\
\text { moments, median, and } \\
\text { Power Spectral Density } \\
\text { (PSD), Feature Change } \\
\text { Ratio (FCR) }\end{array}$ & $\begin{array}{c}\text { PCA, KNN-based } \\
\text { undersampling }\end{array}$ & SVM & \begin{tabular}{|c} 
Sensitivity $=100 \%$ \\
Average false \\
alarm rate per hour \\
$=0.13$ \\
G-mean $=0.97$ \\
F-measure $=0.90$
\end{tabular} \\
\hline 2015 & $\begin{array}{l}\text { P. Fergus et al. } \\
{[36]}\end{array}$ & CHB-MIT & $\begin{array}{l}\text { Delta, theta, alpha, } \\
\text { and beta signal } \\
\text { bands filtering }\end{array}$ & $\begin{array}{c}\text { Peak Frequency, Median } \\
\text { Frequency, variance, root } \\
\text { mean squares, sample } \\
\text { entropy, skewness, and } \\
\text { kurtosis }\end{array}$ & $\begin{array}{c}\text { Linear } \\
\text { discriminant } \\
\text { analysis } \\
\text { backward search }\end{array}$ & $\begin{array}{c}\text { LDC, QDC, UDC, } \\
\text { POLYC, LOGLC, } \\
\text { KNCC, TREEC, } \\
\text { PARZENC, SVC }\end{array}$ & \begin{tabular}{|c|} 
Best results with \\
KNNC: \\
Sensitivity $=84 \%$ \\
Specificity $=85 \%$ \\
AUC $=91 \%$ \\
Global error $=15 \%$ \\
\end{tabular} \\
\hline 2015 & $\begin{array}{l}\text { Cristian Donos } \\
\text { et al. [37] }\end{array}$ & $\begin{array}{l}\text { European } \\
\text { Epilepsy } \\
\text { Database }\end{array}$ & $\mathrm{N} / \mathrm{M}$ & $\begin{array}{c}\text { mean, mean absolute } \\
\text { deviation, variance, } \\
\text { skewness, kurtosis, } \\
\text { autocorrelation, line length, } \\
\text { power, power ratio }\end{array}$ & $\mathrm{N} / \mathrm{M}$ & $\begin{array}{l}\text { Random forest } \\
\text { classifier }\end{array}$ & \begin{tabular}{|c|} 
Mean sensitivity $=$ \\
$93.84 \%$ \\
Mean detection \\
delays $=3.03 \mathrm{~s}$ \\
False detections per \\
hour $=0.33 / \mathrm{h}$ mean \\
\end{tabular} \\
\hline 2015 & $\begin{array}{l}\text { Zisheng Zhang } \\
\text { et al. }[38]\end{array}$ & $\begin{array}{l}\text { Kaggle - } \\
\text { American } \\
\text { Epilepsy } \\
\text { Society } \\
\text { Seizure } \\
\text { Prediction } \\
\text { Challenge }\end{array}$ & $\mathrm{N} / \mathrm{M}$ & $\begin{array}{c}\text { PSD features: Relative } \\
\text { Spectral Powers, Spectral } \\
\text { Power Ratios }\end{array}$ & CART & SVM & $\begin{array}{c}\text { Sensitivity }=100 \% \\
\text { Mean False } \\
\text { Positive }(\mathrm{FP}) \text { rate }= \\
0.073 \mathrm{FP} / \text { hour } \\
\text { Mean prediction } \\
\text { horizon }=58 \\
\text { minutes } \\
\text { AUC }=0.979 \\
\end{array}$ \\
\hline 2016 & $\begin{array}{c}\text { Bruno Direito et } \\
\text { al. }[39]\end{array}$ & $\begin{array}{l}\text { European } \\
\text { Epilepsy } \\
\text { Database }\end{array}$ & $\begin{array}{c}\text { Butterworth Infinite } \\
\text { Impulse } \\
\text { Response (IIR) } \\
\text { filter to remove the } \\
\text { noise }\end{array}$ & \begin{tabular}{|c|} 
AR Modeling predictive \\
error, Decorrelation time, \\
Energy \\
Hjorth, Spectral power, \\
Spectral edge, Energy \\
wavelet coefficients, Mean, \\
Variance \\
Skewness, Kurtosis \\
\end{tabular} & $\mathrm{N} / \mathrm{M}$ & SVM & $\begin{array}{c}\text { false predictions } \\
\text { per hour }=0.20 \\
\text { sensitivity }= \\
38.47 \%\end{array}$ \\
\hline 2016 & $\begin{array}{c}\text { Lung-Chang Lin } \\
\text { et al. }[40]\end{array}$ & $\begin{array}{l}\text { Real-time } \\
\text { personal } \\
\text { dataset }\end{array}$ & $\begin{array}{l}\text { EEG epoch } \\
\text { acquisition }\end{array}$ & \begin{tabular}{|c|} 
Autoregressive modeling \\
predictive error, \\
Decorrelation time, Energy, \\
Entropy, Hjorth, Relative \\
power, Spectral edge, \\
Statistics, Energy of the \\
wavelet coefficients
\end{tabular} & $\begin{array}{c}\text { Correlation based } \\
\text { Feature Selection } \\
(\mathrm{CFS}) \text { approach }\end{array}$ & SVM & \begin{tabular}{|c|} 
Correctness $=$ \\
$97.50 \%$, \\
Sensitivity $=$ \\
$96.92 \%$, \\
Specificity $=$ \\
$97.78 \%$, \\
Precision $=95.45 \%$
\end{tabular} \\
\hline 2016 & $\begin{array}{c}\text { Khurram I. Qazi } \\
\text { et al. [41] }\end{array}$ & $\begin{array}{l}\text { Real-time } \\
\text { personal } \\
\text { dataset }\end{array}$ & $\begin{array}{c}\text { Seizure, pre-seizure } \\
\text { and seizure-free } \\
\text { labelling }\end{array}$ & $\begin{array}{c}\text { Energy (E), range (R), } \\
\text { standard deviation (SD), the } \\
\text { sum of absolute values } \\
\text { (SAV), mean absolute } \\
\text { values (MAV) and variance } \\
\text { (Var) }\end{array}$ & $\mathrm{N} / \mathrm{M}$ & \begin{tabular}{|l|} 
SVM, ANN with \\
supervised \\
learning, K means \\
clustering \\
combined with \\
unsupervised \\
learning \\
algorithms
\end{tabular} & $\begin{array}{c}\text { Accuracy }=85- \\
90 \%\end{array}$ \\
\hline
\end{tabular}




\begin{tabular}{|c|c|c|c|c|c|c|c|}
\hline Year & $\begin{array}{l}\text { Research } \\
\text { Group }\end{array}$ & Dataset Used & Preprocessing & Feature Extraction & $\begin{array}{c}\text { Feature } \\
\text { Selection }\end{array}$ & Classifier Used & Performance \\
\hline 2017 & $\begin{array}{c}\text { Syed } \\
\text { Muhammad } \\
\text { Usman et al. }[4]\end{array}$ & CHB-MIT & $\begin{array}{c}\text { Surrogate channel } \\
\text { creation, Empirical } \\
\text { Mode } \\
\text { Decomposition }\end{array}$ & $\begin{array}{c}\text { Statistical features of time- } \\
\text { domain and spectral } \\
\text { features of frequency } \\
\text { domain }\end{array}$ & $\mathrm{N} / \mathrm{M}$ & $\begin{array}{c}\text { Support Vector } \\
\text { Machine, Naïve } \\
\text { Bayes, K nearest } \\
\text { neighbor }\end{array}$ & $\begin{array}{c}\text { Best results with } \\
\text { SVM: } \\
\text { Sensitivity }= \\
92.23 \% \text { and } \\
\text { average prediction } \\
\text { time }=23.61 \\
\text { minutes }\end{array}$ \\
\hline 2017 & $\begin{array}{c}\text { Han-Tai Shiao } \\
\text { [42] }\end{array}$ & $\begin{array}{l}\text { Mayo Clinic } \\
\text { dataset }\end{array}$ & $\mathrm{N} / \mathrm{M}$ & Spectral features & $\mathrm{N} / \mathrm{M}$ & SVM & $\begin{array}{c}\text { Sensitivity }= \\
90-100 \% \\
\text { Average False } \\
\text { positive rate per } \\
\text { day }=0-0.3\end{array}$ \\
\hline 2018 & $\begin{array}{c}\text { Yanli Yang et al. } \\
\text { [43] }\end{array}$ & $\begin{array}{l}\text { European } \\
\text { Epilepsy } \\
\text { Database }\end{array}$ & $\begin{array}{l}\text { Filtering and } \\
\text { sampling }\end{array}$ & Permutation entropy & $\mathrm{N} / \mathrm{M}$ & SVM & $\begin{array}{c}\text { Average sensitivity } \\
=94 \% \\
\text { False prediction } \\
\text { rates per } \\
\text { hour(FPRh) }= \\
0.111 \\
\text { Average prediction } \\
\text { horizon }=61.93 \mathrm{~min}\end{array}$ \\
\hline 2019 & $\begin{array}{c}\text { Amirhossein } \\
\text { Ahmadi et al. } \\
\text { [44] }\end{array}$ & CHB-MIT & $\mathrm{N} / \mathrm{M}$ & Shannon entropy & $\begin{array}{l}\text { Statistical one- } \\
\text { sample t-test }\end{array}$ & SVM,KNN & $\begin{array}{c}\text { Sensitivity }=83.8 \% \\
(\text { SVM \& KNN) } \\
\text { Specificity }=71 \% \\
\text { (SVM), 67.8 } \\
(\text { KNN) }\end{array}$ \\
\hline 2019 & $\begin{array}{c}\text { Xiashuang Wang } \\
\text { et al. [45] }\end{array}$ & $\begin{array}{l}\text { Bonn } \\
\text { University } \\
\text { Dataset }\end{array}$ & $\begin{array}{l}\text { Digital filtering, } \\
\text { removing artefacts, } \\
\text { re-referencing, and } \\
\text { baseline corrections } \\
\end{array}$ & $\begin{array}{c}\text { Fourier transform, } \\
\text { multitaper spectral analysis, } \\
\text { PACF, and STFT }\end{array}$ & $\mathrm{N} / \mathrm{M}$ & $\begin{array}{l}\text { Random forest } \\
\text { algorithm based } \\
\text { on grid search } \\
\text { optimization }\end{array}$ & $\begin{array}{c}\text { Accuracy }=96.7 \% \\
\text { AUC }=99.0 \%\end{array}$ \\
\hline 2019 & $\begin{array}{c}\text { Yuxing Wang et } \\
\text { al. }[46]\end{array}$ & CHB-MIT & $\begin{array}{c}\text { Pre-ictal state } \\
\text { partition }\end{array}$ & $\begin{array}{c}\text { Wavelet Packet } \\
\text { Decomposition (WPD) }\end{array}$ & $\mathrm{N} / \mathrm{M}$ & Random forest & Accuracy $=84.8 \%$ \\
\hline
\end{tabular}

Table 2. Comparative analysis of the deep learning approach.

\begin{tabular}{|c|c|c|c|c|c|c|c|}
\hline Year & Research Group & Dataset Used & Preprocessing & $\begin{array}{c}\text { Feature } \\
\text { Extraction }\end{array}$ & $\begin{array}{l}\text { Feature } \\
\text { Selection }\end{array}$ & Classifier Used & Performance \\
\hline 2016 & $\begin{array}{c}\text { Mohammad-Parsa Hosseini } \\
\text { et al. }[17]\end{array}$ & $\begin{array}{c}\text { The University of } \\
\text { Pennsylvania and the } \\
\text { Mayo Clinic }\end{array}$ & $\begin{array}{l}\text { Dimensionality } \\
\text { reduction }\end{array}$ & $\mathrm{N} / \mathrm{M}$ & $\mathrm{N} / \mathrm{M}$ & $\begin{array}{l}\text { Stacked auto } \\
\text { encoder }\end{array}$ & $\begin{array}{c}\text { Accuracy }=0.94 \\
\text { Precision }=0.95 \\
\text { Sensitivity }=0.93 \\
\text { False Positive Rate }= \\
0.05 \\
\text { False Negative Rate } \\
=0.06\end{array}$ \\
\hline 2017 & Sachin Talathi [16] & $\begin{array}{c}\text { Bonn University } \\
\text { database }\end{array}$ & $\mathrm{N} / \mathrm{M}$ & $\mathrm{N} / \mathrm{M}$ & $\mathrm{N} / \mathrm{M}$ & \begin{tabular}{|c|} 
Gated Recurrent \\
Unit (GRU) RNNs \\
for seizure \\
detection \\
\end{tabular} & $\begin{array}{l}\text { Accuracy }=100 \% \\
\text { Sensitivity }=98 \%\end{array}$ \\
\hline 2017 & $\begin{array}{c}\text { U. Rajendra Acharya et al. } \\
{[47]}\end{array}$ & $\begin{array}{c}\text { Bonn University } \\
\text { database }\end{array}$ & Normalization & $\mathrm{N} / \mathrm{M}$ & $\mathrm{N} / \mathrm{M}$ & $\mathrm{CNN}$ & $\begin{array}{c}\text { Accuracy }=88.67 \% \\
\text { Sensitivity }=95 \% \\
\text { Specificity }=90 \%\end{array}$ \\
\hline 2017 & Haidar Khan et al. [48] & $\begin{array}{l}\text { 1) Mount Sinai } \\
\text { Epilepsy Center } \\
\text { dataset } \\
\text { 2) CHB-MIT }\end{array}$ & $\mathrm{N} / \mathrm{M}$ & $\mathrm{N} / \mathrm{M}$ & $\mathrm{N} / \mathrm{M}$ & $\begin{array}{l}\text { Convolutional } \\
\text { filters on the } \\
\text { wavelet } \\
\text { transformation }\end{array}$ & $\begin{array}{c}\text { Sensitivity }=87.8 \% \\
\text { False prediction rate } \\
\quad=0.142 / \mathrm{h}\end{array}$ \\
\hline 2018 & $\begin{array}{c}\text { Isabell Kiral-Kornek et al. } \\
{[20]}\end{array}$ & Cook et al.,2013 & $\begin{array}{l}\text { Spectrogram } \\
\text { generation }\end{array}$ & $\mathrm{N} / \mathrm{M}$ & $\mathrm{N} / \mathrm{M}$ & $\begin{array}{l}\text { Deep neural } \\
\text { network }\end{array}$ & $\begin{array}{c}\text { Mean sensitivity }= \\
69 \% \\
\text { Mean time in } \\
\text { warning }=27 \%\end{array}$ \\
\hline 2018 & $\begin{array}{c}\text { Matthias Eberlein et al. } \\
{[19]}\end{array}$ & Kaggle & $\mathrm{N} / \mathrm{M}$ & $\mathrm{N} / \mathrm{M}$ & $\mathrm{N} / \mathrm{M}$ & $\mathrm{CNN}$ & $\mathrm{AUC}=0.73$ \\
\hline
\end{tabular}




\begin{tabular}{|c|c|c|c|c|c|c|c|}
\hline Year & Research Group & Dataset Used & Preprocessing & $\begin{array}{c}\text { Feature } \\
\text { Extraction }\end{array}$ & $\begin{array}{c}\text { Feature } \\
\text { Selection }\end{array}$ & Classifier Used & Performance \\
\hline 2018 & $\begin{array}{c}\text { David Ahmedt-Aristizabal } \\
\text { et al. }[21]\end{array}$ & $\begin{array}{l}\text { Bonn University } \\
\text { database }\end{array}$ & $\mathrm{N} / \mathrm{M}$ & $\mathrm{N} / \mathrm{M}$ & $\mathrm{N} / \mathrm{M}$ & LSTM & $\begin{array}{c}\text { Accuracy }=95.54 \% \\
\text { Sensitivity }=91.83 \\
\text { Specificity }=90.50 \\
\text { Precision }=91.50 \\
\text { AUC }=0.9582\end{array}$ \\
\hline 2018 & $\begin{array}{c}\text { Nhan Duy Truonga et al. } \\
{[49]}\end{array}$ & $\begin{array}{c}\text { CHB-MIT, European } \\
\text { Epilepsy Database, } \\
\text { Kaggle }\end{array}$ & $\begin{array}{c}\text { Short-time Fourier } \\
\text { transform, Windowing }\end{array}$ & $\mathrm{N} / \mathrm{M}$ & $\mathrm{N} / \mathrm{M}$ & $\mathrm{CNN}$ & $\begin{array}{c}\text { Sensitivity }=81.4 \%- \\
81.2 \%-75 \% \\
\text { False prediction rate } \\
=0.06 / \mathrm{h}, 0.16 / \mathrm{h}, \\
0.21 / \mathrm{h}\end{array}$ \\
\hline 2018 & Mengni Zhou et al. [50] & $\begin{array}{c}\text { European Epilepsy } \\
\text { Database, CHB-MIT }\end{array}$ & $\begin{array}{l}\text { Spectrogram } \\
\text { generation }\end{array}$ & $\mathrm{N} / \mathrm{M}$ & $\mathrm{N} / \mathrm{M}$ & $\mathrm{CNN}$ & $\begin{array}{c}\text { Accuracy }= \\
93-97.5 \%\end{array}$ \\
\hline 2018 & R. Schirrmeister et al. [15] & $\begin{array}{c}\text { The Temple } \\
\text { University Hospital } \\
\text { (TUH) EEG } \\
\text { Abnormal } \\
\text { Corpus }\end{array}$ & $\begin{array}{l}\text { Artefact removal and } \\
\text { resampling }\end{array}$ & $\mathrm{N} / \mathrm{M}$ & $\mathrm{N} / \mathrm{M}$ & $\mathrm{CNN}$ & $\begin{array}{c}\text { Accuracy }= \\
84.5-85.4 \% \\
\text { Sensitivity = } \\
75.1-77.3 \% \\
\text { Specificity = } \\
90.5-94.1 \% \\
\text { Accuracy = } \\
81.7-82.5 \%\end{array}$ \\
\hline 2019 & Xinghua Yao et al. [51] & CHB-MIT & $\begin{array}{l}\text { Bidirectional Long } \\
\text { Short-Term Memory } \\
\text { (BiLSTM) }\end{array}$ & $\mathrm{N} / \mathrm{M}$ & $\mathrm{N} / \mathrm{M}$ & Softmax function & $\begin{array}{c}\text { Sensitivity }=87 \% \\
\text { Specificity }=88.60 \% \\
\text { Precision }=88.63 \%\end{array}$ \\
\hline 2019 & Xinghua Yao et al. [23] & CHB-MIT & $\begin{array}{l}\text { Independently } \\
\text { recurrent neural } \\
\text { network (IndRNN) }\end{array}$ & $\mathrm{N} / \mathrm{M}$ & $\mathrm{N} / \mathrm{M}$ & $\mathrm{N} / \mathrm{M}$ & $\begin{array}{l}\text { Sensitivity }=87.3 \% \\
\text { Specificity }=86.7 \% \\
\text { Precision }=87.08 \% \\
\text { F1 score }=87.07 \%\end{array}$ \\
\hline 2019 & Ali Emamia et al. [27] & $\begin{array}{c}\text { NTT Medical Center } \\
\text { Tokyo }\end{array}$ & $\begin{array}{l}\text { 2-D image } \\
\text { construction }\end{array}$ & $\mathrm{N} / \mathrm{M}$ & $\mathrm{N} / \mathrm{M}$ & $\mathrm{CNN}$ & $\begin{array}{c}\text { Median of detected } \\
\text { seizure rate by } \\
\text { minutes }=100 \% \\
\text { False alarm } 0.2 \text { per } \\
\text { hour }\end{array}$ \\
\hline 2019 & Ibrahim Aliyu et al. [52] & $\begin{array}{c}\text { Bonn University } \\
\text { database }\end{array}$ & $\begin{array}{c}\text { Discrete } \\
\text { wavelet transform }\end{array}$ & & & RNN-LSTM & Accuracy $=99 \%$ \\
\hline 2019 & Chien-Liang Liu et al. [53] & Kaggle, CHB-MIT & $\begin{array}{l}\text { PCA, FFT, and data } \\
\text { augmentation }\end{array}$ & $\mathrm{N} / \mathrm{M}$ & $\mathrm{N} / \mathrm{M}$ & Multi-view CNN & $\begin{array}{c}\mathrm{AUC}=0.84 \\
\text { (Kaggle), 0.82-0.89 } \\
(\mathrm{CHB}-\mathrm{MIT})\end{array}$ \\
\hline 2020 & Fabio Pisano et al. [54] & $\begin{array}{c}\text { European Epilepsy } \\
\text { Database }\end{array}$ & $\begin{array}{c}\text { Manual channel } \\
\text { selection, EEG } \\
\text { segmentation and data } \\
\text { augmentation }\end{array}$ & $\mathrm{N} / \mathrm{M}$ & $\mathrm{N} / \mathrm{M}$ & $\mathrm{CNN}$ & $\begin{array}{c}\text { Accuracy }=96.39 \% \\
\text { Specificity }=96.81 \% \\
\text { Sensitivity }=93.20 \% \\
\text { Gmean }= \\
89.92-98.83 \%\end{array}$ \\
\hline
\end{tabular}

Table 3. Comparative analysis of the signal processing approach.

\begin{tabular}{|c|c|c|c|c|c|c|c|}
\hline Year & Research Group & Dataset Used & Preprocessing & Feature Extraction & Feature Selection & Classifier Used & Performance \\
\hline 2012 & $\begin{array}{c}\text { James R. } \\
\text { Williamson et al. } \\
{[55]}\end{array}$ & $\begin{array}{l}\text { European } \\
\text { Epilepsy } \\
\text { Database }\end{array}$ & $\begin{array}{l}\text { Filtering and } \\
\text { normalization }\end{array}$ & $\begin{array}{c}\text { High-dimensional } \\
\text { feature vectors are } \\
\text { extracted from } \\
\text { space-delay covariance } \\
\text { and correlation matrices }\end{array}$ & $\mathrm{N} / \mathrm{M}$ & SVM & $\begin{array}{c}\text { Sensitivity }= \\
95-86 \% \\
\text { AUC }=0.973\end{array}$ \\
\hline 2012 & $\begin{array}{c}\text { Mojtaba } \\
\text { Bandarabadi et } \\
\text { al. }[56]\end{array}$ & $\begin{array}{l}\text { European } \\
\text { Epilepsy } \\
\text { Database }\end{array}$ & $\begin{array}{l}\text { Filtering and } \\
\text { windowing }\end{array}$ & $\begin{array}{c}\text { Normalized spectral } \\
\text { power features, relative } \\
\text { features using bi-variate } \\
\text { approach }\end{array}$ & $\begin{array}{c}\text { normalized } \\
\text { difference of the } \\
\text { percentiles }\end{array}$ & SVM & $\begin{array}{c}\text { Sensitivity }= \\
76.09 \% \\
\text { False positive rate } \\
\text { per hour }=0.15 \\
\text { No. of sleeted } \\
\text { features }=8.75 \\
\text { Seizure occurrence } \\
\text { period }=31.6 \text { min }\end{array}$ \\
\hline 2013 & $\begin{array}{c}\text { Sun-Hee Kim et } \\
\text { al. }[57]\end{array}$ & $\begin{array}{c}\text { Bonn } \\
\text { University } \\
\text { Dataset }\end{array}$ & $\mathrm{N} / \mathrm{M}$ & $\begin{array}{l}\text { Detection of special } \\
\text { characteristics }\end{array}$ & $\mathrm{N} / \mathrm{M}$ & $\begin{array}{c}\text { coercively adjusted } \\
\text { auto regression } \\
\text { (CA-AR) }\end{array}$ & $\begin{array}{l}\text { Root mean square } \\
\quad \text { error }=0.029\end{array}$ \\
\hline
\end{tabular}


(Table 3) contd.....

\begin{tabular}{|c|c|c|c|c|c|c|c|}
\hline Year & Research Group & Dataset Used & Preprocessing & Feature Extraction & Feature Selection & Classifier Used & Performance \\
\hline 2013 & $\begin{array}{c}\text { Yang Zheng et al. } \\
{[58]}\end{array}$ & $\begin{array}{l}\text { European } \\
\text { Epilepsy } \\
\text { Database }\end{array}$ & $\begin{array}{c}\text { Artefact removal } \\
\text { and filtering }\end{array}$ & $\begin{array}{c}\text { Bivariate empirical } \\
\text { mode decomposition, } \\
\text { Mean Phase Coherence } \\
\text { (MPC) }\end{array}$ & $\begin{array}{c}\text { A quantitative } \\
\text { method based on the } \\
\text { seizure prediction } \\
\text { characteristic } \\
\text { was proposed for the } \\
\text { feature selection }\end{array}$ & $\begin{array}{l}\text { The preictal } \\
\text { changes of the } \\
\text { MPC time courses } \\
\text { were used to raise } \\
\text { the seizure alarms }\end{array}$ & $\begin{array}{c}\text { Sensitivity }= \\
70-80 \% \text { with } \\
\text { Seizure Prediction } \\
\text { Horizon }=10 \text { min } \\
\text { and False Prediction } \\
\text { Rate }(\max )=0.15 \\
\mathrm{FP} / \mathrm{h} .\end{array}$ \\
\hline 2014 & $\begin{array}{c}\text { Zhen Zhang et al. } \\
{[59]}\end{array}$ & $\begin{array}{c}\text { Real-time } \\
\text { personal } \\
\text { dataset }\end{array}$ & $\begin{array}{l}\text { Optimal channel } \\
\text { selection }\end{array}$ & Approximate entropy & $\mathrm{N} / \mathrm{M}$ & $\mathrm{N} / \mathrm{M}$ & $\begin{array}{c}\text { Prediction accuracy } \\
\quad=94.59 \% \\
\text { False prediction rate } \\
=0.084 / \mathrm{h} \\
\text { Mean prediction } \\
\text { time }=26.64 \mathrm{~min}\end{array}$ \\
\hline 2014 & $\begin{array}{c}\text { Nilufer Ozdemir } \\
\text { et al. [60] }\end{array}$ & $\begin{array}{l}\text { European } \\
\text { Epilepsy } \\
\text { Database }\end{array}$ & $\begin{array}{l}\text { Artefact Removal } \\
\text { and Segmentation }\end{array}$ & $\begin{array}{c}\text { Hilbert Huang } \\
\text { Transform, total energy }\end{array}$ & $\begin{array}{l}\text { Filtering feature } \\
\text { selection and } \\
\text { Correlation-based } \\
\text { Feature Selection } \\
\text { (CFS) with } \\
\text { the best first search } \\
\text { algorithm }\end{array}$ & Bayesian network & $\begin{array}{c}\text { Sensitivity }= \\
96.55 \% \\
\text { Mean detection } \\
\text { latency }=33.21 \\
\text { False positives per } \\
\text { hour }(\mathrm{FPs} / \mathrm{h})=0.21 \\
\text { Time spent in } \\
\text { warning }(\mathrm{FP} \%)= \\
13.896 \mathrm{~min}\end{array}$ \\
\hline 2015 & $\begin{array}{c}\text { Hamidreza } \\
\text { Namazi et al. } \\
{[61]}\end{array}$ & $\begin{array}{l}\text { Real-time } \\
\text { personal } \\
\text { dataset }\end{array}$ & Filtering & $\begin{array}{l}\text { Hurst exponent and } \\
\text { fractal dimension }\end{array}$ & $\mathrm{N} / \mathrm{M}$ & $\mathrm{N} / \mathrm{M}$ & $\begin{array}{l}\text { Seizure occurrence } \\
\text { period }=25.76 \\
\text { seconds }\end{array}$ \\
\hline 2015 & $\begin{array}{c}\text { Kohtaroh } \\
\text { Edakawa et al. } \\
{[62]}\end{array}$ & $\begin{array}{l}\text { Real-time } \\
\text { personal } \\
\text { dataset }\end{array}$ & $\mathrm{N} / \mathrm{M}$ & $\begin{array}{l}\text { Phase-amplitude } \\
\text { coupling } \\
\text { (PAC) }\end{array}$ & $\begin{array}{c}\theta \text {-high } \gamma, \\
\alpha \text {-high } \gamma, \\
\beta \text {-high } \gamma, \\
\theta \text { phase of } 10-80 \mathrm{~Hz} \\
\text { amplitude, } \\
\text { High } \gamma \text { amplitude } \\
\text { alone }\end{array}$ & $\begin{array}{l}\text { Synchronisation } \\
\text { index (SI) }\end{array}$ & $\begin{array}{c}\text { Sensitivity }=100, \\
\text { False detection rate } \\
\text { per hour }=0.713\end{array}$ \\
\hline 2016 & $\begin{array}{c}\text { A. Sharmila et al. } \\
{[63]}\end{array}$ & $\begin{array}{c}\text { Bonn } \\
\text { University } \\
\text { Dataset }\end{array}$ & $\mathrm{N} / \mathrm{M}$ & \begin{tabular}{|c|} 
Statistical features from \\
Discrete Wavelet \\
Transform (DWT)
\end{tabular} & $\mathrm{N} / \mathrm{M}$ & $\begin{array}{l}\text { Naïve Bayes, K- } \\
\text { nearest neighbor }\end{array}$ & $\begin{array}{c}\text { Accuracy }=100 \% \\
\text { (with Naïve Bayes) }\end{array}$ \\
\hline 2016 & $\begin{array}{c}\text { Mark H. Myers et } \\
\text { al. }[64]\end{array}$ & CHB-MIT & Filtering & $\begin{array}{l}\text { Phase Locking } \\
\text { Thresholds }\end{array}$ & $\mathrm{N} / \mathrm{M}$ & $\mathrm{N} / \mathrm{M}$ & $\begin{array}{c}\text { Sensitivity }=77 \% \\
\text { Precision }=88 \% \\
\text { False positive per } \\
\text { hour }=0.17 \\
\end{array}$ \\
\hline 2017 & $\begin{array}{c}\text { Turky N. } \\
\text { Alotaiby et al. } \\
{[65]}\end{array}$ & CHB-MIT & $\mathrm{N} / \mathrm{M}$ & $\begin{array}{c}\text { Common Spatial Pattern } \\
\text { (CSP) }\end{array}$ & $\mathrm{N} / \mathrm{M}$ & $\begin{array}{c}\text { Linear discriminant } \\
\text { analysis (LDA) }\end{array}$ & $\begin{array}{c}\text { Average sensitivity } \\
=0.89 \\
\text { Average specificity } \\
=0.37 \\
\text { Average False } \\
\text { Prediction Rate }= \\
0.39 \\
\text { Average prediction } \\
\text { time }=68.71 \\
\text { minutes }\end{array}$ \\
\hline 2017 & $\begin{array}{l}\text { Amirmasoud } \\
\text { Ahmadi et al. } \\
{[66]}\end{array}$ & $\begin{array}{c}\text { Bonn } \\
\text { University } \\
\text { Dataset } \\
\end{array}$ & $\mathrm{N} / \mathrm{M}$ & $\begin{array}{l}\text { Wavelet packets } \\
\text { transform }\end{array}$ & $\begin{array}{l}\text { Standard Deviation, } \\
\text { Root mean square }\end{array}$ & $\begin{array}{l}\text { SVM with the } \\
\text { radial basis } \\
\text { function }\end{array}$ & Accuracy $=97.85 \%$ \\
\hline 2018 & $\begin{array}{c}\text { Ahmed I. Sharaf } \\
\text { et al. [67] }\end{array}$ & $\begin{array}{l}\text { Bonn } \\
\text { University } \\
\text { Dataset }\end{array}$ & $\begin{array}{c}\text { Tunable Q- } \\
\text { Wavelet } \\
\text { Transformation } \\
\text { (TQWT) }\end{array}$ & \begin{tabular}{|c|} 
Chaotic features, \\
statistical features, \\
power spectrum \\
features, Co-occurrence \\
matrix
\end{tabular} & Firefly algorithm & Random forest & $\begin{array}{c}\text { Accuracy }=99 \% \\
\text { Precision }=97 \% \\
\text { Specificity }=97 \% \\
\text { Recall }=98 \% \\
\text { F-measure }=98 \% \\
\text { Matthew's } \\
\text { correlation } \\
\text { coefficient }=95 \%\end{array}$ \\
\hline 2019 & $\begin{array}{c}\text { Naghmeh } \\
\text { Mahmoodian et } \\
\text { al. }[68]\end{array}$ & $\begin{array}{l}\text { European } \\
\text { Epilepsy } \\
\text { Database }\end{array}$ & $\begin{array}{l}\text { Filtering and } \\
\text { windowing }\end{array}$ & Cross-bispectral analysis & $\mathrm{N} / \mathrm{M}$ & SVM & $\begin{array}{c}\text { Sensitivity }=100 \% \\
\text { False positive rate } \\
(\mathrm{FPR})=0.044 \\
\text { Prediction time }= \\
51-96 \text { minutes }\end{array}$ \\
\hline
\end{tabular}


(Table 3) contd.....

\begin{tabular}{|c|c|c|c|c|c|c|c|}
\hline Year & Research Group & Dataset Used & Preprocessing & Feature Extraction & Feature Selection & Classifier Used & Performance \\
\hline 2019 & $\begin{array}{l}\text { Agustina Garcés } \\
\text { Correa et al. [69] }\end{array}$ & CHB-MIT & $\begin{array}{l}\text { Adaptive filter } \\
\text { and signal } \\
\text { averaging }\end{array}$ & $\mathrm{N} / \mathrm{M}$ & $\mathrm{N} / \mathrm{M}$ & $\mathrm{N} / \mathrm{M}$ & $\begin{array}{c}\text { Sensitivity }= \\
90.29 \% \\
\text { Specificity }=73.7 \%\end{array}$ \\
\hline 2019 & $\begin{array}{c}\text { Hafeez A. } \\
\text { Agboola et al. } \\
{[70]}\end{array}$ & CHB-MIT & $\mathrm{N} / \mathrm{M}$ & $\begin{array}{c}\text { Low-level feature } \\
\text { extraction }\end{array}$ & $\begin{array}{l}\text { High level feature } \\
\text { extraction }\end{array}$ & SVM, ANN & $\begin{array}{c}\text { Sensitivity }= \\
87.26 \%(\mathrm{SVM}), \\
75.5 \%(\mathrm{ANN}) \\
\text { False alarm per } \\
\text { hour }=0.09(\mathrm{SVM}), \\
0.13(\mathrm{ANN}) \\
\text { Seizure occurrence } \\
\text { period }=31 \mathrm{~min} \\
(\mathrm{SVM}), 29 \mathrm{~min} \\
(\mathrm{ANN})\end{array}$ \\
\hline
\end{tabular}

Table 4. Comparative analysis of the hybrid approach.

\begin{tabular}{|c|c|c|c|c|c|c|c|}
\hline Year & $\begin{array}{l}\text { Research } \\
\text { Group }\end{array}$ & Dataset used & Preprocessing & Feature Extraction & Feature selection & Classifier used & Performance \\
\hline 2005 & $\begin{array}{c}\text { Nihal Fatma } \\
\text { Guler et al. } \\
\text { [71] }\end{array}$ & $\begin{array}{l}\text { Bonn } \\
\text { University } \\
\text { database }\end{array}$ & $\mathrm{N} / \mathrm{M}$ & Lyapunov exponents & $\mathrm{N} / \mathrm{M}$ & RNN & $\begin{array}{c}\text { Sensitivity }= \\
96.88-96.13 \% \\
\text { Specificity }= \\
97.38 \% \\
\text { Accuracy = } \\
96.79 \%\end{array}$ \\
\hline 2015 & $\begin{array}{c}\text { Khalid } \\
\text { Abualsaud et } \\
\text { al. }[72]\end{array}$ & $\begin{array}{l}\text { Bonn } \\
\text { University } \\
\text { database }\end{array}$ & $\begin{array}{c}\text { Compressive } \\
\text { Sensing (CS), } \\
\text { Discrete Cosine } \\
\text { Transform } \\
\text { (DCT) }\end{array}$ & DWT & $\mathrm{N} / \mathrm{M}$ & $\begin{array}{l}\text { Noise-aware Signal } \\
\text { Combination (NSC) } \\
\text { ensemble classifier }\end{array}$ & $\begin{array}{c}\text { Accuracy }=80 \% \\
\text { (for } \mathrm{SNR}=1 \mathrm{~dB} \text { ), } \\
84 \% \text { (for } \\
\mathrm{SSNR}=5 \mathrm{~dB} \text { ), } 88 \% \\
\text { (for } \mathrm{SNR}=10 \mathrm{~dB} \text { ) }\end{array}$ \\
\hline 2018 & \begin{tabular}{|c|} 
Kostas M. \\
Tsiouris et al. \\
{$[73]$}
\end{tabular} & CHB-MIT & Segmentation & $\begin{array}{l}\text { Cross-correlation, time } \\
\text { domain, frequency } \\
\text { domain, graph theory }\end{array}$ & $\mathrm{N} / \mathrm{M}$ & LSTM & $\begin{array}{c}\text { Sensitivity }= \\
99.84 \% \\
\text { Specificity }= \\
99.86 \% \\
\text { FPR per hour }= \\
0.02 \\
\text { Preictal duration }= \\
120\end{array}$ \\
\hline 2018 & $\begin{array}{c}\text { Punjal Agarwa } \\
\text { et al. }[74]\end{array}$ & Kaggle & $\begin{array}{l}\text { Image sampling, } \\
\text { dimensionality } \\
\text { reduction }\end{array}$ & $\mathrm{FFT}, \mathrm{CNN}$ & $\mathrm{N} / \mathrm{M}$ & Hybrid CNN-SVM & $\begin{array}{c}\text { Accuracy }= \\
97.07 \% \\
\text { Sensitivity = } \\
96.47 \% \\
\text { Specificity = } \\
98.81 \%\end{array}$ \\
\hline 2018 & $\begin{array}{c}\text { Farrikh Alzami } \\
\text { et al. }[75]\end{array}$ & $\begin{array}{l}\text { Bonn } \\
\text { University } \\
\text { database }\end{array}$ & $\mathrm{N} / \mathrm{M}$ & DWT & $\begin{array}{c}\text { rank-aggregation } \\
\text { (RA) }\end{array}$ & $\begin{array}{l}\text { Adaptive hybrid feature } \\
\text { selection-based ensemble }\end{array}$ & $\begin{array}{c}\text { Accuracy = } \\
96-100 \% \\
\text { Sensitivity = } \\
96.58-100 \% \\
\text { Specificity = } \\
97.47-100 \%\end{array}$ \\
\hline 2018 & $\begin{array}{l}\text { J.B. Schiratti } \\
\text { et al. }[76]\end{array}$ & $\begin{array}{c}\text { European } \\
\text { Epilepsy } \\
\text { Database }\end{array}$ & Down sampling & $\begin{array}{c}\text { Time and frequency } \\
\text { domain features }\end{array}$ & $\mathrm{N} / \mathrm{M}$ & $\begin{array}{l}\text { Logistic regression, } \\
\text { Weighted Ensemble } \\
\text { (WE) classifier }\end{array}$ & $\begin{array}{c}\text { ROC AUC score }= \\
0.87\end{array}$ \\
\hline 2018 & $\begin{array}{l}\text { Lal Hussain } \\
\text { [77] }\end{array}$ & $\begin{array}{l}\text { Bonn } \\
\text { University } \\
\text { database }\end{array}$ & $\begin{array}{l}\text { Wavelet threshold } \\
\text { demonising } \\
\text { method, } \\
\text { Daudechies (db4) } \\
\text { wavelet, PCA }\end{array}$ & $\begin{array}{c}\text { Time domain, } \\
\text { frequency domain and } \\
\text { complexity }\end{array}$ & $\mathrm{N} / \mathrm{M}$ & $\begin{array}{l}\text { SVM, KNN, Decision } \\
\text { tree, Ensemble }\end{array}$ & $\begin{array}{c}\text { Accuracy }=99.5 \% \\
\text { (with SVM) } \\
\text { AUC }=0.9991 \\
\text { (with SVM) }\end{array}$ \\
\hline 2018 & $\begin{array}{c}\text { Debdeep } \\
\text { Sikdar et al. } \\
\text { [78] }\end{array}$ & $\begin{array}{l}\text { Bonn } \\
\text { University } \\
\text { database }\end{array}$ & $\begin{array}{l}\text { Wavelet-based } \\
\text { decomposition }\end{array}$ & $\begin{array}{c}\text { Multifractal Detrended } \\
\text { Fluctuation Analysis } \\
\text { (MF-DFA) }\end{array}$ & $\mathrm{N} / \mathrm{M}$ & SVM & $\begin{array}{c}\text { Accuracy }=99.6 \% \\
\text { Precision }=99.3 \% \\
\text { Recall }=99.3 \% \\
\text { Specificity }=99.7 \\
\text { F-score }=99.3\end{array}$ \\
\hline
\end{tabular}




\begin{tabular}{|c|c|c|c|c|c|c|c|}
\hline Year & $\begin{array}{l}\text { Research } \\
\text { Group }\end{array}$ & Dataset used & Preprocessing & Feature Extraction & Feature selection & Classifier used & Performance \\
\hline 2019 & $\begin{array}{c}\text { Hisham Daoud } \\
\text { et al. }[22]\end{array}$ & CHB-MIT & $\mathrm{N} / \mathrm{M}$ & $\mathrm{N} / \mathrm{M}$ & $\mathrm{N} / \mathrm{M}$ & $\begin{array}{c}\text { MLP } \\
\text { DCNN+MLP } \\
\text { DCNN+Bi-LSTM } \\
\text { DCAE+Bi-LSTM } \\
\text { DCAE+Bi-LSTM+CS }\end{array}$ & $\begin{array}{c}\text { Sensitivity }= \\
99.72 \% \\
\text { Specificity }= \\
99.60 \% \\
\text { Accuracy }= \\
99.60 \% \\
\text { False Alarm per } \\
\text { hour }=0.004 \\
\text { Prediction time }=1 \\
\text { hour }\end{array}$ \\
\hline 2019 & $\begin{array}{c}\text { Omer Turk et } \\
\text { al. }[79]\end{array}$ & $\begin{array}{c}\text { Bonn } \\
\text { University } \\
\text { database }\end{array}$ & $\mathrm{N} / \mathrm{M}$ & $\begin{array}{c}\text { Continuous } \\
\text { Wavelet Transform } \\
(\mathrm{CWT})\end{array}$ & Resize image & $\mathrm{CNN}$ & $\begin{array}{l}\text { Accuracy }= \\
90.50-100 \%\end{array}$ \\
\hline 2020 & $\begin{array}{c}\text { Yunyuan Gao } \\
\text { et al. }[80]\end{array}$ & CHB-MIT & Signal denoising & $\begin{array}{l}\text { Power spectrum } \\
\text { density analysis }\end{array}$ & $\mathrm{N} / \mathrm{M}$ & $\begin{array}{l}\text { Inception-v3, ResNet152, } \\
\text { Inception-ResNet-v2 }\end{array}$ & $\begin{array}{c}\text { Accuracy }=92.6 \% \\
\text { Sensitivity }= \\
97.1 \% \\
\text { Preictal duration } \\
\text { (Minutes) }=30\end{array}$ \\
\hline
\end{tabular}

\section{DATASETS}

Table $\mathbf{5}$ shows the comparative analysis of datasets used by various researchers for epileptic seizure detection and prediction. The datasets considered are highly unlike each other. The differences are in terms of EEG recording mechanism used i.e., sEEG or iEEG, number of subjects used, number of channels used, duration of the recordings, and number of recordings with the true positive case. The ' $*$ ' in the Table 5 suggests that the parameter has not been specified by the researchers. The research is also diversified in intra-subject (subject-specific), and inter-subject (adaptive) approaches. The selection of the dataset is highly dependent on these parameters. As derived from Table 5, majorly used datasets in the field of epileptic seizure detection/prediction are CHB-MIT [81, 82], Kaggle competition dataset [83], Bonn university dataset [84], and BCI competition dataset [85]. Apart from these, available significant datasets are the TUH dataset [86], European epilepsy dataset [87], and EEG epilepsy dataset [88]. Based on the subject-specific or adaptive approach of epileptic seizure prediction, the selection of the dataset is done [89]. For reproducibility of the results, utilization of the dataset must be clearly mentioned.

Table 5. Epilepsy seizure prediction datasets used by different researchers.

\begin{tabular}{|c|c|c|c|c|}
\hline References & Dataset & Type of EEG & No. of Subjects & No. of Channels \\
\hline Syed Muhammad Usman et al. [4] & CHB-MIT & sEEG & 22 & 23 \\
\hline Pouya Bashivan et al. [14] & Collected by authors & sEEG & 13 & 64 \\
\hline Robin Tibor Schirrmeister et al. [15] & BCI competition-IV & sEEG & $*$ & $*$ \\
\hline Sachin Talati et al. [16] & Bonn University & sEEG & $*$ & $128 /$ single \\
\hline Mohammad-Parsa Hosseini et al. [17] & BCI competition & iEEG & 9 & 15 \\
\hline Mohammad-Parsa Hosseini et al. [18] & Kaggle competition & iEEG & $\begin{array}{l}5 \text { Dog and } 2 \\
\text { Human }\end{array}$ & $\begin{array}{l}16 \text { and varying } \\
\text { channels }\end{array}$ \\
\hline Matthias Eberlein et al. [19] & Kaggle competition & iEEG & $\begin{array}{l}5 \text { Dog and } 2 \\
\text { Human }\end{array}$ & $\begin{array}{l}16 \text { and varying } \\
\text { channels }\end{array}$ \\
\hline Isabell Kiral-Kornek et al. [20] & Cook et al. [89] & iEEG & 10 & 16 \\
\hline David Ahmedt-Aristizabal et al. [21] & Bonn University & sEEG & $*$ & 128/Single \\
\hline Hisham Daoud et al. [22] & CHB-MIT & sEEG & 22 & $18 / 23$ \\
\hline Xinghua Yao et al. [23] & CHB-MIT & sEEG & 22 & $18 / 23$ \\
\hline Nick Hershey et al. [24] & Stanford hospital and Lucile Packard Children Hospital & sEEG \& iEEG & $1,36,363$ & $3-142$ \\
\hline Ghulam Muhammad et al. [25] & CHB-MIT & sEEG & 22 & $18 / 23$ \\
\hline Xiaoyan Wei et al. [26] & $\begin{array}{c}\text { Xinjiang } \\
\text { Medical } \\
\text { University }\end{array}$ & sEEG & 13 & $*$ \\
\hline Ali Emamia et al. [27] & NTT Medical Center Tokyo & sEEG & 16 & 19 \\
\hline Turky N. Alotaiby et al. [65] & CHB-MIT & sEEG & 22 & $18 / 23$ \\
\hline P. Fergus et al. [90] & CHB-MIT & sEEG & 22 & $18 / 23$ \\
\hline Lung-Chang Lin et al. [40] & $\begin{array}{l}\text { Kaohsiung } \\
\text { Medical } \\
\text { University } \\
\text { Hospital }\end{array}$ & sEEG & 5 & 21 \\
\hline
\end{tabular}


(Table 5) contd.....

\begin{tabular}{|c|c|c|c|c|}
\hline References & Dataset & Type of EEG & No. of Subjects & No. of Channels \\
\hline Julius Hulsmann [91] & PhysioNet & sEEG & 109 & 64 \\
\hline Benjamin H. Brinkmann [92] & Kaggle competition & $\mathrm{iEEG}$ & 8 & 16 \\
\hline Ricardo Aler [93] & BCI-III competition & sEEG & 3 & 32 \\
\hline Ning Wang et al. [34] & Freiburg dataset & iEEG & 21 & 128 \\
\hline Xinghua Yao et al. [51] & CHB-MIT & sEEG & 22 & $18 / 23$ \\
\hline Nipun Dilesh Perera et al. [94] & CHB-MIT & sEEG & 22 & 23 \\
\hline Fayas Asharindavida et al. [95] & CHB-MIT, EPILEPSIAE-purchased & sEEG \& iEEG & $22 / 125$ & $22 / 217$ \\
\hline Yinxia Liu et al. [96] & Bonn University & sEEG & $*$ & 128/Single \\
\hline Gurwinder Singh et al. [97] & Bonn University & sEEG & $*$ & $128 /$ Single \\
\hline I. Omerhodzic et al. [98] & Bonn University & sEEG & * & 128/Single \\
\hline Punjal Agarwal et al. [74] & Kaggle competition & iEEG & $\begin{array}{c}5 \text { Dog and } 2 \\
\text { Human }\end{array}$ & $\begin{array}{c}16 \text { and varying } \\
\text { channels }\end{array}$ \\
\hline Omer Turk et al. [79] & Bonn University & sEEG & $*$ & 128/Single \\
\hline Debdeep Sikdara et al. [78] & Bonn University & sEEG & * & 128/Single \\
\hline Kostas M. Tsiouris et al. [73] & CHB-MIT & sEEG & 22 & 23 \\
\hline Maarten Larmuseau et al. [99] & Kaggle competition & iEEG & 4 Dog & 16 \\
\hline Kaat Vandecasteele et al. $[100]$ & Hospital ECG, Wearable ECG, Wearable PPG & * & 11 & 1 \\
\hline Ehsan Dadgar-kiani et al. [101] & Kaggle competition & iEEG & 1 & 16 \\
\hline M. Stella Mercy [102] & Bonn University & iEEG & 2 & 1 \\
\hline Sharanreddy et al. [103] & CHB-MIT & sEEG & 22 & 23 \\
\hline U. Rajendra Acharya et al. [47] & Bonn University & sEEG & $*$ & 128/Single \\
\hline Ibrahim Aliyu et al. [52] & Bonn University & sEEG & $*$ & $128 /$ Single \\
\hline
\end{tabular}

\section{TOOLS AND LIBRARIES USED FOR IMPLE- MENTATION}

Though an ample amount of work has already been done in the field of epileptic seizure detection and prediction, the available published work does not contain much information related to the implementation environment used by the researchers. Table $\mathbf{6}$ summarizes the details available in past work on epileptic seizure detection and prediction.

It can be derived from Table 6 that most of the work is implemented using Matlab or Python. Various popular tools provided by Matlab are EEGLAB [104], Brainstorm [105], FieldTrip [106], EEGVIS [107], NFT [104], and BCILAB [104]. Python provides different libraries like MNE [108], MNE-Python [109], PyEEG [110], and Pyprep [111]. Whereas $R$ provides packages like eegkit [112], eegUtils [113], eegR [114], erpR [115], and ERP [116]. While undertaking any research project which includes EEG signal analysis, researchers spent an ample amount of time in finalizing the platform to adapt for implementation. Following detail shows a comparison of Matlab, Python, and R concerning various parameters useful for implementation:

Table 6. Frameworks used by various researchers for epileptic seizure prediction.

\begin{tabular}{|c|c|}
\hline References & Framework Used \\
\hline Syed Muhammad Usman et al. [4] & Matlab \\
\hline Pouya Bashivan et al. [14] & Lasagne \\
\hline Mohammad-Parsa Hosseini et al. [18] & Pytorch \\
\hline Xiaoyan Wei et al. [26] & Python with Tensorflow \\
\hline Ali Emami et al. [27] & Python \\
\hline Lung-Chang Lin et al. [40] & Weka \\
\hline Julius Hu"lsmann et al. [91] & Numpy, Scipy, MNE \\
\hline Benjamin H. Brinkmann et al. [92] & LibSVM \\
\hline Ricardo Aler et al. [93] & Weka \\
\hline Ning Wang et al. [34] & Matlab spiderbox toolbox \\
\hline Fayas Asharindavida et al. [95] & Matlab \\
\hline Maarten Larmuseau [99] & Sci-kit learn, keras \\
\hline Ehsan Dadgar-Kiani et al. [101] & Sci-kit learn, keras \\
\hline M. Stella Mercy [102] & LibSVM \\
\hline Sharanreddy et al. [103] & Matlab \\
\hline U. Rajendra Acharya et al. [47] & Matlab \\
\hline Ibrahim Aliyu et al. [52] & Python with Tensorflow and Keras \\
\hline
\end{tabular}


Open Source: The major advantage of using Python and $\mathrm{R}$ over Matlab is that they are open source, making them quite attractive solution to many applications. Applications developed in Python and R can be widely distributable, making it easier to enable collaboration between scientists at various locations. Maturity of Tools: The tools supported by Matlab are quite mature to use compared to Python and R. It is best suitable for detailed analysis of the EEG signals. Flexibility of Manipulation: The maturity makes it less flexible to manipulate and extend features in Matlab. Community Support: Community support has important significance in selecting the specific platform for research. Matlab and Python both have huge community support when it comes to EEG signal processing. This area is quite evolving in R; however, the statistical analysis performed by $\mathrm{R}$ is no comparable.

One of the best solutions could be to use the best feature of each platform and use it in integration. Python has an Oct2py library [117], which converts Python data structures to Matlab or Octave data structures and vice versa. It is the simplest and most stable way to run Matlab functions on Python, and most EEGLAB functions may be called from within python using this method. R provides the reticulate package [118], which allows running Python code directly within $\mathrm{R}$.

\section{CONCLUSION}

EEG-based epilepsy detection and prediction using a machine learning approached has taken a boost following the technology evolution. The essential aspects of EEG waveformbased research for epileptic seizure detection and prediction have been discussed in detail. The comparative analysis of various datasets and implementation platforms considered in the past approaches along with details of traditional machine learning, deep learning, and combination of both - the hybrid approach is given, which would help novice to begin. The selection of dataset and implementation environment is highly dependent on requirement of the research. Various criteria play an important role, such as for datasets: subject-specific research or generalized research to be carried out, number of channels to consider, number of patients to consider; and for implementation environment: budget, programming expertize, and size of the dataset. Shortcomings of epileptic seizure prediction approaches are also discussed to shed light on future enhancements.

\section{CONSENT FOR PUBLICATION}

Not applicable.

\section{FUNDING}

None.

\section{CONFLICT OF INTEREST}

The authors declare no conflict of interest, financial or otherwise.

\section{ACKNOWLEDGEMENTS}

Declared none.

\section{REFERENCES}

[1] I. Osorio, Hitten P. Zaveri, Mark G. Frei, and Susan Arthurs, Epilepsy: the intersection of neurosciences, biology, mathematics, engineering, and physics., CRC press, 2016.

[2] C. MD, "What is epilepsy?", WebMD. Available at: https://www.webmd.com/epilepsy/ understanding-epilepsy-basics\#1

[3] E. MD, and P. RN, "Types of Seizures", Epilepsy Foundation, 2021.[Accessed: 17- Jun- 2021]. Available at: https://www.epilepsy.com/learn/types-seizures

[4] S. Usman, M. Usman, and S. Fong, "Epileptic seizures prediction using machine learning methods", Comput. Math. Meth. Med., vol. 2017, pp. 1-10, 2017.

[http://dx.doi.org/10.1155/2017/9074759]

[5] C. Guerreiro, "Epilepsy: Is there hope?", Indian J. Med. Res., vol. 144 no. 5 , pp. $657-660,2016$.

[http://dx.doi.org/10.4103/ijmr.IJMR_1051_16] [PMID: 28361817]

[6] A. Roman-Gonzalez, EEG Signal Processing for BCI Applications.Advan. Intelli. Soft Comp., 2012, pp. 571-591. [http://dx.doi.org/10.1007/978-3-642-23187-2_36]

[7] B. Schelter, J. Timmer, and A. Schulze-Bonhage, Seizure prediction in epilepsy., Wiley-VCH: Hoboken, 2008. [Imprint] [http://dx.doi.org/10.1002/9783527625192]

[8] K-Y. Jung, Analysis of eeg in medically intractable epilepsy.Bio. Med. Phys., Biomedical Engineering, Springer, 2018, pp. 177-192. [http://dx.doi.org/10.1007/978-981-13-0908-3 8]

[9] M. Iwasaki, K. Jin, N. Nakasato, and T. Tominaga, "Noninvasive evaluation for epilepsy surgery", Neuro. Medi-chirur., vol. 56, no. 10, pp. 632-640, 2016.

[http://dx.doi.org/10.2176/nmc.ra.2016-0186]

[10] M. Mula, and H.R. Cock, "More than seizures: improving the lives of people with refractory epilepsy", Eur. J. Neurol., vol. 22, no. 1, pp. 24-30, 2015.

[http://dx.doi.org/10.1111/ene.12603] [PMID: 25367637]

[11] C. Im, Computational EEG Analysis., $1^{\text {st }}$ ed. Springer, 2018. [http://dx.doi.org/10.1007/978-981-13-0908-3]

[12] J. Satheesh Kumar, and P. Bhuvaneswari, "Analysis of Electroencephalography (EEG) Signals and Its Categorization-A Study", Proced. Eng., vol. 38, pp. 2525-2536, 2012.

[http://dx.doi.org/10.1016/j.proeng.2012.06.298]

[13] Saeid Sanei and Jonathon A Chambers, EEG signal processing., John Wiley \& Sons, 2013.

[14] P. Bashivan, I. Rish, M. Yeasin, and N. Codella, "Learning representations from eeg with deep recurrent-convolutional neural networks", arXiv preprint arXiv:1511.06448, 2015.

[15] Robin Tibor Schirrmeister, Lukas Gemein, Katharina Eggensperger, Frank Hutter, and Tonio Ball, "Deep learning with convolutional neural networks for decoding and visualization of EEG pathology", CoRR, abs/1708.08012, 2017.

[16] S. Sachin, "Deep recurrent neural networks for seizure detection and early seizure detection systems", arXiv preprint arXiv:1706.03283, 2017.

[17] M-P. Hosseini, H. Soltanian-Zadeh, K. Elisevich, and D. Pompili, Cloud-based deep learning of big eeg data for epileptic seizure prediction. In 2016 IEEE global conference on signal and information processing (GlobalSIP)., IEEE, 2016, pp. 1151-1155.

[http://dx.doi.org/10.1109/GlobalSIP.2016.7906022]

[18] M-P. Hosseini, D. Pompili, K. Elisevich, and H.S. Zadeh, "Optimized deep learning for eeg big data and seizure prediction bci via internet of things", IEEE Transactions on Big Data, vol. 3, no. 4, pp. 392-404, 2017.

[http://dx.doi.org/10.1109/TBDATA.2017.2769670]

[19] M. Eberlein, R. Hildebrand, R. Tetzlaff, N. Hoffmann, L. Kuhlmann, B. Brinkmann, and J. Mu"ller, "Convolutional neural networks for epileptic seizure prediction", 2018 IEEE International Conference on Bioinformatics and Biomedicine (BIBM), 2018 pp. 2577-2582 IEEE, Spain

[http://dx.doi.org/10.1109/BIBM.2018.8621225]

[20] I. Kiral-Kornek, S. Roy, E. Nurse, B. Mashford, P. Karoly, T. Carroll, D. Payne, S. Saha, S. Baldassano, T. O'Brien, D. Grayden, M. Cook, D. Freestone, and S. Harrer, "Epileptic seizure prediction using big data and deep learning: toward a mobile system", EBioMedicine, vol. 27, pp. 103-111, 2018.

[http://dx.doi.org/10.1016/j.ebiom.2017.11.032] [PMID: 29262989]

[21] D. Ahmedt-Aristizabal, C. Fookes, K. Nguyen, and S. Sridharan, "Deep classification of epileptic signals", 2018 40th Annual International Conference of the IEEE Engineering in Medicine and Biology Society (EMBC), 2018 pp. 332-335 IEEE, United States [http://dx.doi.org/10.1109/EMBC.2018.8512249]

[22] H. Daoud, and M.A. Bayoumi, "Bayoumi. Efficient epileptic seizure prediction based on deep learning", IEEE Trans. Biomed. Circuits 
Syst., vol. 13, no. 5, pp. 804-813, 2019.

[http://dx.doi.org/10.1109/TBCAS.2019.2929053] [PMID: 31331897]

[23] X. Yao, Q. Cheng, and G-Q. Zhang, "Qiang Cheng, and Guo-Qiang Zhang. A novel independent rnn approach to classification of seizures against non-seizures", arXiv preprint arXiv:1903.09326, 2019.

[24] Nick Hershey, Detecting epileptic seizures in electroencephalogram.

[25] Muhammad G., Masud M., Amin S.U., and Alrobaea R., "and Mohammed F Alhamid. Automatic seizure detection in a mobile multimedia framework", IEEE Access, vol. 6, pp. 45372-45383, 2018. [http://dx.doi.org/10.1109/ACCESS.2018.2859267]

[26] X. Wei, L. Zhou, Z. Chen, L. Zhang, and Y. Zhou, "Automatic seizure detection using three-dimensional CNN based on multi-channel EEG", BMC Med. Inform. Decis. Mak., vol. 18, no. 5, suppl. Suppl. 5, p. 111, 2018.

[http://dx.doi.org/10.1186/s12911-018-0693-8] [PMID: 30526571]

[27] A. Emami, N. Kunii, T. Matsuo, T. Shinozaki, K. Kawai, and H. Takahashi, "Seizure detection by convolutional neural network-based analysis of scalp electroencephalography plot images", Neuroimage Clin., vol. 22, 2019.101684

[http://dx.doi.org/10.1016/j.nicl.2019.101684] [PMID: 30711680]

[28] P. Geethanjali, Fundamentals of brain signals and its medical application usingdata analysis techniques.Computational Intelligence for Big Data Analysis., Springer, 2015, pp. 181-197.

[http://dx.doi.org/10.1007/978-3-319-16598-1_8]

[29] V. Patel, S. Buch, and A. Ganatra, "A review on eeg based epileptic seizure prediction using machine learning techniques", International Conference on Intelligent Computing, Information and Control Systems, 2019 pp. 384-391 Springer, 2019

[30] Eeg signal processing: Theory and applications.Neural Engineering., Springer, 2013, pp. 259-303.

[http://dx.doi.org/10.1007/978-1-4614-5227-0 5]

[31] Y. Roy, H. Banville, I. Albuquerque, A. Gramfort, T.H. Falk, and J. Faubert, "Deep learning-based electroencephalography analysis: a systematic review", J. Neural Eng., vol. 16, no. 5, 2019.051001 [http://dx.doi.org/10.1088/1741-2552/ab260c] [PMID: 31151119]

[32] A. Craik, Y. He, and J.L. Contreras-Vidal, "Deep learning for electroencephalogram (EEG) classification tasks: a review", J. Neural Eng., vol. 16, no. 3, 2019.031001

[http://dx.doi.org/10.1088/1741-2552/ab0ab5] [PMID: 30808014]

[33] T. Netoff, Y. Park, and K. Parhi, "Seizure prediction using cost sensitive support vector machine", 2009 Annual International Conference of the IEEE Engineering in Medicine and Biology Society, 2009 pp. 3322-3325 IEEE, 2009

[http://dx.doi.org/10.1109/IEMBS.2009.5333711]

[34] N. Wang, and M.R. Lyu, "Lyu. Extracting and selecting distinctive eeg features for efficient epileptic seizure prediction", IEEE J. Biomed. Health Inform., vol. 19, no. 5, pp. 1648-1659, 2015. [http://dx.doi.org/10.1109/JBHI.2014.2358640] [PMID: 25248205]

[35] P. Ghaderyan, A. Abbasi, and M.H. Sedaaghi, "An efficient seizure prediction method using KNN-based undersampling and linear frequency measures", J. Neurosci. Methods, vol. 232, pp. 134-142, 2014.

[http://dx.doi.org/10.1016/j.jneumeth.2014.05.019] [PMID: 24875624]

[36] Paul Fergus, David Hignett, Abir Hussain, Dhiya Al-Jumeily, and Khaled AbdelAziz, "Automatic epileptic seizure detection using scalp eeg and advanced artificial intelligence techniques", BioMed research international, vol. 2015, 2015 .

[http://dx.doi.org/10.1155/2015/986736]

[37] C. Donos, M. Dümpelmann, and A. Schulze-Bonhage, "Early seizure detection algorithm based on intracranial eeg and random forest classification", Int. J. Neural Syst., vol. 25, no. 5, 2015.1550023 [http://dx.doi.org/10.1142/S0129065715500239] [PMID: 26022388]

[38] Z. Zhang, and K. Keshab, "Seizure prediction using polynomial svm classification", 2015 37th Annual International Conference of the IEEE Engineering in Medicine and Biology Society (EMBC), 2015 pp. 5748-5751 IEEE, 2015

[39] B. Direito, C.A. Teixeira, F. Sales, M. Castelo-Branco, and A. Dourado, "A realistic seizure prediction study based on multiclass svm", Int. J. Neural Syst., vol. 27, no. 3, 2017.1750006 [http://dx.doi.org/10.1142/S012906571750006X] [PMID: 27873554]

[40] L-C. Lin, S.C-J. Chen, C-T. Chiang, H-C. Wu, R.C. Yang, and C.S. Ouyang, "ReiCheng Yang, and Chen-Sen Ouyang. Classification preictal and interictal stages via integrating interchannel and timedomain analysis of eeg features", Clin. EEG Neurosci., vol. 48, no. 2, pp. 139-145, 2017.

[http://dx.doi.org/10.1177/1550059416649076] [PMID: 27177554]

[41] I. Khurram, "Qazi, Hak-Keung Lam, Bo Xiao, Gaoxiang Ouyang, and
Xunhe Yin. Classification of epilepsy using computational intelligence techniques", CAAI Transactions on Intelligence Technology, vol. 1, no. 2, pp. 137-149, 2016.

[http://dx.doi.org/10.1016/j.trit.2016.08.001]

[42] H-T. Shiao, V. Cherkassky, J. Lee, B. Veber, E.E. Patterson, B.H. Brinkmann, and G.A. Worrell, "Patterson, Benjamin H Brinkmann, and Gregory A Worrell. Svm-based system for prediction of epileptic seizures from ieeg signal", IEEE Trans. Biomed. Eng., vol. 64, no. 5, pp. 1011-1022, 2017.

[http://dx.doi.org/10.1109/TBME.2016.2586475] [PMID: 27362758]

[43] Y. Yang, M. Zhou, Y. Niu, C. Li, R. Cao, B. Wang, P. Yan, Y. Ma, and J. Xiang, "PengfeiYan, Yao Ma, and Jie Xiang. Epileptic seizure prediction based on permutation entropy", Front. Comput. Neurosci., vol. 12 , p. $55,2018$.

[http://dx.doi.org/10.3389/fncom.2018.00055] [PMID: 30072886]

[44] A. Ahmadi, and H. Soltanian-Zadeh, "Epileptic seizure prediction using spectral entropy-based features of eeg", 2019 4th International Conference on Pattern Recognition and Image Analysis (IPRIA), 2019 pp. 124-129 IEEE, 2019

[http://dx.doi.org/10.1109/PRIA.2019.8785984]

[45] X. Wang, G. Gong, N. Li, and S. Qiu, "Detection analysis of epileptic eeg using a novel random forest model combined with grid search optimization", Front. Hum. Neurosci., vol. 13, p. 52, 2019.

[http://dx.doi.org/10.3389/fnhum.2019.00052] [PMID: 30846934]

[46] Y. Wang, J. Cao, X. Lai, and D. Hu, "Epileptic state classification for seizure prediction with wavelet packet features and random forest", 2019 Chinese Control And Decision Conference (CCDC), , 2019pp. 3983-3987

[http://dx.doi.org/10.1109/CCDC.2019.8833249]

[47] U.R. Acharya, S.L. Oh, Y. Hagiwara, J.H. Tan, and H. Adeli, "Deep convolutional neural network for the automated detection and diagnosis of seizure using EEG signals", Comput. Biol. Med., vol. 100, pp. $270-278,2018$.

[http://dx.doi.org/10.1016/j.compbiomed.2017.09.017]

[PMID: 28974302]

[48] H. Khan, L. Marcuse, M. Fields, K. Swann, and B. Yener, "Focal onset seizure prediction using convolutional networks", IEEE Trans. Biomed. Eng., vol. 65, no. 9, pp. 2109-2118, 2018. [http://dx.doi.org/10.1109/TBME.2017.2785401] [PMID: 29989952]

[49] N.D. Truong, A.D. Nguyen, L. Kuhlmann, M.R. Bonyadi, J. Yang, S Ippolito, and O. Kavehei, "Convolutional neural networks for seizure prediction using intracranial and scalp electroencephalogram", Neural Netw., vol. 105, pp. 104-111, 2018.

[http://dx.doi.org/10.1016/j.neunet.2018.04.018] [PMID: 29793128]

[50] M. Zhou, C. Tian, R. Cao, B. Wang, Y. Niu, T. Hu, H. Guo, and J. Xiang, "andJie Xiang. Epileptic seizure detection based on eeg signals and cnn", Front. Neuroinform., vol. 12, p. 95, 2018. [http://dx.doi.org/10.3389/fninf.2018.00095] [PMID: 30618700]

[51] X. Yao, X. Li, Q. Ye, Y. Huang, Q. Cheng, and G-Q. Zhang, "A robust deep learning approach for automatic classification of seizures against non-seizures", arXiv preprint arXiv:1812.06562, 2018.

[52] I. Aliyu, Y.B. Lim, and C.G. Lim, "Epilepsy detection in eegsignal using recurrent neural network", Proceedings of the 2019 3rd International Conference on Intelligent Systems, Metaheuristics \& Swarm Intelligence, 2019pp. 50-53

[http://dx.doi.org/10.1145/3325773.3325785]

[53] C-L. Liu, B. Xiao, W-H. Hsaio, and S. Vincent, "Tseng. Epileptic seizure prediction with multi-view convolutional neural networks", IEEE Access, vol. 7, pp. 170352-170361, 2019.

[http://dx.doi.org/10.1109/ACCESS.2019.2955285]

[54] Fabio Pisano, Giuliana Sias, Alessandra Fanni, and Barbara Cannas, "Convolutional neural network for seizure detection of nocturnal frontal lobe epilepsy", Complexity, vol. 2020, 2020.

[55] J.R. Williamson, D.W. Bliss, D.W. Browne, and J.T. Narayanan, "Seizure prediction using EEG spatiotemporal correlation structure", Epilepsy Behav., vol. 25, no. 2, pp. 230-238, 2012.

[http://dx.doi.org/10.1016/j.yebeh.2012.07.007] [PMID: 23041171]

[56] M. Bandarabadi, B.D. Cesar A Teixeira, and A. Dourado, "Epileptic seizure prediction based on a bivariate spectral power methodology", 2012 Annual International Conference of the IEEE Engineering in Medicine and Biology Society, , 2012pp. 5943-5946 [http://dx.doi.org/10.1109/EMBC.2012.6347347]

[57] Sun-Hee Kim, Christos Faloutsos, and Hyung-Jeong Yang, "Coercively adjustedauto regression model for forecasting in epilepsy eeg", Computational and mathematical methods in medicine, vol. 2013, 2013.

[58] Y. Zheng, G. Wang, K. Li, G. Bao, and J. Wang, "Epileptic seizure 
prediction using phase synchronization based on bivariate empirical mode decomposition", Clin. Neurophysiol., vol. 125, no. 6, pp. 1104-1111, 2014.

[http://dx.doi.org/10.1016/j.clinph.2013.09.047] [PMID: 24296277]

[59] Z. Zhang, Z. Chen, Y. Zhou, S. Du, Y. Zhang, T. Mei, and X. Tian, "Construction of rules for seizure prediction based on approximate entropy", Clin. Neurophysiol., vol. 125, no. 10, pp. 1959-1966, 2014. [http://dx.doi.org/10.1016/j.clinph.2014.02.017] [PMID: 24690391]

[60] Nilufer Ozdemir, and Esen Yildirim, "Patient specific seizure prediction system using hilbert spectrum and bayesian networks classifiers", Computational and mathematical methods in medicine, vol. 2014, 2014 [http://dx.doi.org/10.1155/2014/572082]

[61] H. Namazi, V.V. Kulish, J. Hussaini, J. Hussaini, A. Delaviz, F. Delaviz, S. Habibi, and S. Ramezanpoor, "A signal processing based analysis and prediction of seizure onset in patients with epilepsy", Oncotarget, vol. 7, no. 1, pp. 342-350, 2016.

[http://dx.doi.org/10.18632/oncotarget.6341] [PMID: 26586477]

[62] K. Edakawa, T. Yanagisawa, H. Kishima, R. Fukuma, S. Oshino, H.M. Khoo, M. Kobayashi, M. Tanaka, and T. Yoshimine, "Detection of epileptic seizures using phase-amplitude coupling in intracranial electroencephalography", Sci. Rep., vol. 6, p. 25422, 2016. [http://dx.doi.org/10.1038/srep25422] [PMID: 27147119]

[63] A. Sharmila, and P. Geethanjali, "Dwt based detection of epileptic seizure from eegsignals using naive bayes and k-nn classifiers", IEEE Access, vol. 4, pp. 7716-7727, 2016.

[http://dx.doi.org/10.1109/ACCESS.2016.2585661]

[64] H. Mark, "Myers, Akshay Padmanabha, Gahangir Hossain, Amy L de Jongh Curry, and Charles D Blaha. Seizure prediction and detection via phase and amplitude lock values", Front. Hum. Neurosci., vol. 10, p. $80,2016$.

[65] Alotaiby Turky N, Alshebeili Saleh A, Alotaibi Faisal M, and Alrshoud Saud R, "Epileptic seizure prediction using csp and lda for scalp eeg signals. ", Computational intelligence and neuroscience, vol. 2017, 2017

[66] A. Ahmadi, V. Shalchyan, and M.R. Daliri, A new method for epileptic seizure classification in eeg using adapted wavelet packets.2017 Electric Electronics, Computer Science, Biomedical Engineerings' Meeting (EBBT), IEEE, 2017, pp. 1-4. [http://dx.doi.org/10.1109/EBBT.2017.7956756]

[67] I Ahmed, "An automated approach for epilepsy detection based on tunable q-wavelet and firefly feature selection algorithm", International journal of biomedical imaging, vol. 2018, 2018.

[68] N. Mahmoodian, J. Haddadnia, A. Illanes, and A. Boese, "andMichael Friebe. Seizure prediction with cross-higher-order spectral analysis of eeg signals", Signal Image Video Process., pp. 1-8, 2019.

[69] "Lorena L Orosco, Pablo Diez, and Eric Laciar Leber.Adaptive filtering for epileptic event detection in the eeg", J. Med. Biol. Eng., vol. 39 , no. 6 , pp. $912-918,2019$

[http://dx.doi.org/10.1007/s40846-019-00467-w]

[70] S. David, "Aribike, Afolabi E Lesi, and Alfred A Susu. Seizure prediction with adaptive feature representation learning", ARCHIVOS DE MEDICINA, vol. 10, no. 2, p. 294, 2019.

[71] N.F. Gu"ler, E.D. Ubeyli, and I. Gu"ler, "Recurrent neural networks" employing lyapunov exponents for eeg signals classification", Expert Syst. Appl., vol. 29, no. 3, pp. 506-514, 2005. [http://dx.doi.org/10.1016/j.eswa.2005.04.011]

[72] Khalid Abualsaud, Massudi Mahmuddin, Mohammad Saleh, and Amr Mohamed, "Ensemble classifier for epileptic seizure detection for imperfect eeg data", The Scientific World Journal, vol. 2015, 2015. [http://dx.doi.org/10.1155/2015/945689]

[73] M. Kostas, "Tsiouris, Vasileios C Pezoulas, Michalis Zervakis, Spiros Konitsiotis, Dimitrios D Koutsouris, and Dimitrios I Fotiadis. A long short-term memory deep learning network for the prediction of epileptic seizures using eeg signals", Comput. Biol. Med., vol. 99, pp. 24-37, 2018.

[http://dx.doi.org/10.1016/j.compbiomed.2018.05.019]

[74] P. Agarwal, H-C. Wang, and K. Srinivasan, "Epileptic seizure prediction over eeg data using hybrid cnn-svm model with edge computing services", MATEC Web of Conferences, vol. 210, 2018p. 03016 EDP Sciences.

[http://dx.doi.org/10.1051/matecconf/201821003016]

[75] F. Alzami, J. Tang, Z. Yu, and S. Wu, "CL Philip Chen, Jane You, andJun Zhang. Adaptive hybrid feature selection-based classifier ensemble for epileptic seizure classification", IEEE Access, vol. 6, pp. 29132-29145, 2018.

[http://dx.doi.org/10.1109/ACCESS.2018.2838559]
[76] J-B. Schiratti, "Jean-Eudes Le Douget, Michel Le van Quyen, Slim Essid, and Alexandre Gramfort. An ensemble learning approach to detect epileptic seizures from long intracranial eeg recordings", 2018 IEEE International Conference on Acoustics, Speech and Signal Processing (ICASSP), , 2018pp. 856-860

[http://dx.doi.org/10.1109/ICASSP.2018.8461489]

[77] L. Hussain, "Detecting epileptic seizure with different feature extracting strategies using robust machine learning classification techniques by applying advance parameter optimization approach", Cogn Neurodyn, vol. 12, no. 3, pp. 271-294, 2018. [http://dx.doi.org/10.1007/s11571-018-9477-1] [PMID: 29765477]

[78] D. Sikdar, R. Roy, and M. Mahadevappa, "Epilepsy and seizure characterisation by multifractal analysis of eeg subbands", Biomed. Signal Process. Control, vol. 41, pp. 264-270, 2018.

[http://dx.doi.org/10.1016/j.bspc.2017.12.006]

[79] Ö. Türk, and M.S. Özerdem, "Epilepsy detection by using scalogram" based convolutional neural network from eeg signals", Brain Sci., vol. 9, no. 5, p. $115,2019$.

[http://dx.doi.org/10.3390/brainsci9050115] [PMID: 31109020]

[80] Y. Gao, B. Gao, Q. Chen, J. Liu, and Y. Zhang, "Deep convolutional neural network-based epileptic electroencephalogram (eeg) signal classification", Front. Neurol., vol. 11, p. 375, 2020. [http://dx.doi.org/10.3389/fneur.2020.00375] [PMID: 32528398]

[81] A.H. Shoeb, "'Application of machine learning to epileptic seizure onset detection and treatment."”, Ph.D Thesis, Harvard University, Cambridge, Massachusetts, 2009.

[82] L Ary, "Physiobank, physiotoolkit, and physionet: components of a new research resource for complex physiologic signals", circulation, vol. 101, no. 23, pp. e215-e220, 2000.

[83] "American Epilepsy Society Seizure Prediction Challenge | Kaggle", Kaggle.com, 2015. Available at: https://www.kaggle. com/c/seizureprediction/data

[84] G. Ralph, "Andrzejak, Klaus Lehnertz, Florian Mormann, Christoph Rieke, PeterDavid, and Christian E Elger. Indications of nonlinear deterministic and finite dimensional structures in time series of brain electrical activity: Dependence on recording region and brain state", Phys. Rev. E, vol. 64, no. 6, p. 061907, 2001.

[http://dx.doi.org/10.1103/PhysRevE.64.061907]

[85] "Bci competitions", Bbci.de, 2021.[Accessed: 18- Jun- 2021] Available at: http://www.bbci.de/competition/

[86] I. Obeid, and J. Picone, "The temple university hospital eeg data corpus", Front. Neurosci., vol. 10, p. 196, 2016. [http://dx.doi.org/10.3389/fnins.2016.00196] [PMID: 27242402]

[87] M. Ihle, H. Feldwisch-Drentrup, C.A. Teixeira, A. Witon, B. Schelter, J. Timmer, and A. Schulze-Bonhage, "EPILEPSIAE - a European epilepsy database", Comput. Methods Programs Biomed., vol. 106, no. 3, pp. 127-138, 2012. [http://dx.doi.org/10.1016/j.cmpb.2010.08.011] [PMID: 20863589]

[88] Piyush Swami, Bijaya Panigrahi, Sanjeev Nara, and Manvir Bhatia, Eeg epilepsy datasets, 2016.

[89] M.J. Cook, T.J. O’Brien, S.F. Berkovic, M. Murphy, A. Morokoff, G. Fabinyi, W. D'Souza, R. Yerra, J. Archer, L. Litewka, S. Hosking, P. Lightfoot, V. Ruedebusch, W.D. Sheffield, D. Snyder, K. Leyde, and D. Himes, "Prediction of seizure likelihood with a long-term, implanted seizure advisory system in patients with drug-resistant epilepsy: a first-in-man study", Lancet Neurol., vol. 12, no. 6, pp. 563-571, 2013.

[http://dx.doi.org/10.1016/S1474-4422(13)70075-9]

[PMID: 23642342]

[90] A. Paul Fergus, "Hussain, David Hignett, Dhiya Al-Jumeily, Khaled Abdel-Aziz,and Hani Hamdan. A machine learning system for automated whole-brain seizure detection", Applied Computing and Informatics, vol. 12, no. 1, pp. 70-89, 2016.

[http://dx.doi.org/10.1016/j.aci.2015.01.001]

[91] Julius Hu"1smann, Michal Jirku, and Alexander Dyck, Eeg signal decoding and classification.

[92] B.H. Brinkmann, E.E. Patterson, C. Vite, V.M. Vasoli, D. Crepeau, M. Stead, J.J. Howbert, V. Cherkassky, J.B. Wagenaar, B. Litt, and G.A. Worrell, "Forecasting seizures using intracranial eeg measures and svm in naturally occurring canine epilepsy", PLoS One, vol. 10, no. 8, p. e0133900, 2015.

[http://dx.doi.org/10.1371/journal.pone.0133900] [PMID: 26241907]

[93] R. Aler, S.M. In'e, N. Galva', and M. Jos'e, "Valls. Applying evolution strategiesto preprocessing eeg signals for brain-computer interfaces", Inf. Sci., vol. 215, pp. 53-66, 2012.

[http://dx.doi.org/10.1016/j.ins.2012.05.012]

[94] Nipun Dilesh Perera, Chamara Madarasingha, and De Silva Anjula C, 
"Spatial feature reduction in long-term eeg for patient-specific epileptic seizure event detection", Proceedings of the 9th International Conference on Signal Processing Systems, pp. 230-234, 2017.

[95] F. Asharindavida, "M Shamim Hossain, Azeemsha Thacham, Hedi Khammari,Irfan Ahmed, Fahad Alraddady, and Mehedi Masud. A forecasting tool for prediction of epileptic seizures using a machine learning approach", Concurr. Comput., vol. 32, no. 1, 2020.e5111 [http://dx.doi.org/10.1002/cpe.5111]

[96] Y. Liu, W. Zhou, Q. Yuan, and S. Chen, "Automatic seizure detection using wavelet transform and SVM in long-term intracranial EEG", IEEE Trans. Neural Syst. Rehabil. Eng., vol. 20, no. 6, pp. 749-755, 2012.

[http://dx.doi.org/10.1109/TNSRE.2012.2206054] [PMID: 22868635]

[97] G. Singh, M. Kaur, and D. Singh, "Detection of epileptic seizure using wavelet transformation and spike based features", 2015 2nd International Conference on Recent Advances in Engineering \& Computational Sciences (RAECS), , 2015pp. 1-4

[http://dx.doi.org/10.1109/RAECS.2015.7453376]

[98] I. Omerhodzic, S. Avdakovic, A. Nuhanovic, and K. Dizdarevic, "Amir Nuhanovic, and Kemal Dizdarevic.Energy distribution of eeg signals: Eeg signal wavelet-neural network classifier", rXiv preprint arXiv:1307.7897, 2013

[99] M. Larmuseau, "Epileptic seizure prediction using deep learning", Thesis formaster of science in biomedical engineering, Faculty of Engineering and Architecture - Ghent University, Belgium, 2015-16.

[100] K. Vandecasteele, T. De Cooman, Y. Gu, E. Cleeren, K. Claes, W.V. Paesschen, S.V. Huffel, and B. Hunyadi, "Automated epileptic seizure detection based on wearable ecg and ppg in a hospital environment", Sensors (Basel), vol. 17, no. 10, p. 2338, 2017.

[http://dx.doi.org/10.3390/s17102338] [PMID: 29027928]

[101] Ehsan Dadgar-Kiani, Cagan Alkan, and Ali Shameli, "Applying machine learning for human seizure prediction", Stanford University, Tech. Rep., 2016.

[102] M. Stella Mercy, "Performance analysis of epileptic seizure detection using dwt \&ica with neural networks", International Journal of Computational Engineering Research, vol. 2, no. 4, pp. 1109-1113, 2012.

[103] P. Sharanreddy, and P.K. Kulkarni, "Eeg signal classification for epilepsy seizuredetection using improved approximate entropy", Int $J$ Public Health Sci, vol. 2, no. 1, pp. 23-32, 2013.

[104] A. Delorme, and S. Makeig, "EEGLAB: an open source toolbox for analysis of single-trial EEG dynamics including independent component analysis", J. Neurosci. Methods, vol. 134, no. 1, pp. 9-21, 2004.

[http://dx.doi.org/10.1016/j.jneumeth.2003.10.009] [PMID: 15102499]

[105] S. Baillet, and C. John, "Mosher, Richard M Leahy, and David W
Shattuck. Brainstorm: a matlab toolbox for the processing of meg and eeg signals", Neuroimage, vol. 9, pp. S246-S246, 1999.

[106] Robert Oostenveld, Pascal Fries, Eric Maris, and Jan-Mathijs Schoffelen, "Fieldtrip: open source software for advanced analysis of meg, eeg, and invasive electrophysiological data", Computational intelligence and neuroscience, vol. 2011, 2011.

[http://dx.doi.org/10.1155/2011/156869]

[107] K.A. Robbins, "Robbins. Eegvis: a matlab toolbox for browsing, exploring, and viewing large datasets", Front. Neuroinform., vol. 6, p. $17,2012$.

[http://dx.doi.org/10.3389/fninf.2012.00017] [PMID: 22654753]

[108] L. Esch, C. Dinh, E. Larson, D. Engemann, and M. Jas, "Mne: Software for acquiring, processing, and visualizing meg/eeg data", In: Magnetoencephalography: From Signals to Dynamic Cortical Networks, 2019, pp. 355-371.

[109] A. Gramfort, M. Luessi, E. Larson, D.A. Engemann, D. Strohmeier, C. Brodbeck, R. Goj, M. Jas, T. Brooks, L. Parkkonen, and M. Hämäläinen, "MEG and EEG data analysis with MNE-Python", Front. Neurosci., vol. 7, p. 267, 2013.

[http://dx.doi.org/10.3389/fnins.2013.00267] [PMID: 24431986]

[110] Forrest Sheng Bao, Xin Liu, and Christina Zhang, "Pyeeg: an open source python module for eeg/meg feature extraction", Computational intelligence and neuroscience, vol. 2011, 2011.

[111] ""pyprep"", PyPI, 2020.Accessed: 18- Jun- 2021 Available at: https://pypi.org/project/ pyprep/

[112] Helwig Nathaniel E, and Helwig Maintainer Nathaniel E, Package 'eegkit'., 2018.

[113] Matt Craddock, eegUtils: A collection of utilities for EEG analysis, 2019. R package version 0.3.0.9000.

[114] "'tdeenes/eegR"'", GitHub, 2020.Accessed: 18- Jun- 2021 Available at: https://github.com/tdeenes/eegR/

[115] G Arcara, and A Petrova, "Erp analysis, graphics and utility functions", R Package Version 0.2, 2014.

[116] D. Causeur, C.F. Sheu, M.C. Chu, and F. Rufini, "Erp: Significance analysis of eventrelated potentials data;", http://CRAN.R-project.org/package=ERP.R package version, vol. 1, 2018no. 1, pp. 54-62.

[117] accessed July 27, 2020https://pypi.org/project/ oct2py/ oct2py 5.0.4,

[118] JJ Allaire, Ushey Kevin, and Tang Yuan, reticulate: $R$ Interface to Python, 2017.

[119] T. Maiwald, M. Winterhalder, R. Aschenbrenner-Scheibe, and U. Henning, "Voss, Andreas Schulze-Bonhage, and Jens Timmer. Comparison of three nonlinear seizure prediction methods by means of the seizure prediction characteristic", Physica D, vol. 194, no. 3-4, pp. 357-368, 2004.

[http://dx.doi.org/10.1016/j.physd.2004.02.013]

\section{(C) 2021 Patel et al.}

This is an open access article distributed under the terms of the Creative Commons Attribution 4.0 International Public License (CC-BY 4.0), a copy of which is available at: https://creativecommons.org/licenses/by/4.0/legalcode. This license permits unrestricted use, distribution, and reproduction in any medium, provided the original author and source are credited. 